The Federal Reserve BanK of KANSAS City Research Working Papers

Model Uncertainty and Intertemporal Tax Smoothing

Yulei Luo, Jun Nie and Eric R. Young

January 2012; Revised June 2014

RWP 12-01 


\title{
Model Uncertainty and Intertemporal Tax Smoothing*
}

\author{
Yulei $\mathrm{Luo}^{\dagger}$ \\ The University of Hong Kong \\ Jun $\mathrm{Nie}^{\ddagger}$ \\ Federal Reserve Bank of Kansas City \\ Eric R. Young ${ }^{\S}$ \\ University of Virginia
}

\begin{abstract}
In this paper we examine how model uncertainty due to the preference for robustness (RB) affects optimal taxation and the evolution of debt in the Barro tax-smoothing model (1979). We first study how the government spending shocks are absorbed in the short run by varying taxes or through debt under RB. Furthermore, we show that introducing RB improves the model's predictions by generating (i) the observed relative volatility of the changes in tax rates to government spending, (ii) the observed comovement between government deficits and spending, and (iii) more consistent behavior of government budget deficits in the U.S. economy. Finally, we show that RB can also improve the model's predictions in the presence of multiple shocks.
\end{abstract}

Keywords: Robustness, Model Uncertainty, Taxation Smoothing.

JEL Classification Numbers: D83, E6, H3, H6.

${ }^{*}$ We are grateful for the Editor and an anonymous referee for many constructive suggestions and comments. We also would like to thank Woong Yong Park for his detailed comments, Tasos Karantounias, Ken Kasa, Fred Kwan, Charles Leung, Yang Lu, Tom Sargent, Chris Sims, Tao Zhu, and Xiaodong Zhu for helpful discussions, and seminar and conference participants at Federal Reserve Bank of Kansas City, City University of Hong Kong, Hong Kong University of Science and Technology, the AEA Annual Meeting, and the Midwest Macro Meeting. Luo thanks the General Research Fund (GRF\#: HKU749711) in Hong Kong and the HKU seed funding program for basic research for financial support. Nie thanks Ethan Struby for research assistance. Young thanks the Bankard Fund for Political Economy for financial support. All errors are the responsibility of the authors. The views expressed here are the opinions of the authors only and do not necessarily represent those of the Federal Reserve Bank of Kansas City or the Federal Reserve System.

${ }^{\dagger}$ School of Economics and Finance, Faculty of Business and Economics, The University of Hong Kong, Hong Kong. E-mail: yulei.luo@gmail.com.

${ }^{\ddagger}$ Research Department, The Federal Reserve Bank of Kansas City. E-mail: jun.nie@kc.frb.org.

$\S$ Department of Economics, University of Virginia, Charlottesville, VA 22904. E-mail: ey2d@virginia.edu. 


\section{Introduction}

Figure 1 presents the post-war behavior of US government spending, average tax rates, and government debt. ${ }^{1}$ In this paper we ask how to rationalize the joint behavior of these fiscal variables; specifically, we ask whether the stochastic properties of the joint behavior of these fiscal variables are consistent with the idea that governments seek to use debt to smooth the taxes needed to finance an exogenous stream of government spending.

Barro (1979) proposed a simple full-information rational expectations (FI-RE) tax-smoothing model with only uncontingent debt in which the government spreads the burden of raising distortionary income taxes over time in order to minimize their welfare losses to address these questions. Specifically, the model predicts that the government should issue debt in order to spread the increases in tax rates over longer periods and minimize the welfare losses when facing a positive government spending shock. ${ }^{2}$ Furthermore, the model also predicts that changes in the tax rate are unpredictable, i.e., the tax rate should follow a random walk. ${ }^{3}$ It is worth noting that the taxsmoothing hypothesis (TSH) model is analogous to the permanent income hypothesis (PIH) model in which consumers smooth consumption over time; tax rates respond to permanent changes in the public budgetary burden rather than transitory changes. ${ }^{4}$

Huang and Lin (1993) and Ghosh (1995) went beyond the random-walk tests and use the analysis of saving in Campbell (1987) to test the restrictions on the joint behavior of budget deficits and government spending implied by the tax-smoothing hypothesis. ${ }^{5}$ They found mixed empirical evidence for the TSH. For example, Huang and Lin (1993) applied a log-linearized TSH model to the U.S. data from $1929-1988$ and found that the TSH is rejected for the full sample period, but it is not rejected for the sub-sample period from $1947-1988$. Ghosh (1995) used the US data from 1961 - 1988 and Canadian data from 1962 - 1988 and found that the TSH model cannot be rejected for either country. Cashin, Haque, and Olekalns (2003) found the TSH is rejected by Pakistan data for the period $1954-1995 .{ }^{6}$ Adler (2006) tested the TSH using

\footnotetext{
${ }^{1}$ The data are taken from National Income and Product Accounts (NIPA) and The Economic Report of President, 2011. It is worth pointing out that we are using a measure of average tax rates (defined as tax revenues divided by GDP) rather than marginal tax rates. For a detailed description of the data and definitions, please see Section 4.2 .

${ }^{2}$ The tax-smoothing model is widely used in the literature to address various fiscal policy issues; see Sahasakul (1986), Bohn (1990), Trehan and Walsh (1990), Ghosh (1995), Angeletos (2002), Lloyd-Ellis, Zhan, and Zhu (2002), and Aiyagari et al. (2002). For the tax-smoothing setting with state-contingent debt, see Lucas and Stokey (1983), and Karantounias (2013).

${ }^{3}$ Although Barro $(1979,1981)$ report that in the US data the prediction that the tax rate is a random walk is difficult to reject statistically, Sahasakul (1986) is able to reject it.

${ }^{4}$ For a statement of this equivalence, see Sargent (2001).

${ }^{5}$ It is well known that it is often difficult to reject the null hypothesis of a random walk for many macroeconomic time series given the length of the data. Moreover, as argued in Ghosh (1995), the TSH might be only one of many potential explanations for unpredictable tax rate changes.

${ }^{6}$ More precisely, the tax-smoothing hypothesis is rejected by a Wald test of the parameter restriction at the 5 percent level (as reported in their Table 1); the statements in the paper that suggest that the TSH is not rejected are
} 
the Swedish central government data and found that it is not possible to statistically reject the TSH for the full period 1952 - 1999, but the TSH is rejected using the sub-sample period from 1970 - 1996. Olekalns (1997) investigated Australian data, and again rejects the TSH.

However, as shown in Table 3 in the next section, the standard FI-RE TSH model cannot generate two key stochastic properties of the joint behavior of government deficits, tax rates, and spending: (i) the relative volatility of changes in tax rates and spending and (ii) the contemporaneous correlation between government deficits and spending, in the U.S. economy. Specifically, the model generates too low relative volatility and too high contemporaneous correlation. As a result, we also find that the TSH is rejected in this standard full-information RE framework using the US data.

In this paper, we show that model uncertainty due to the preference for robustness (RB) significantly improves the model's ability to fit the data discussed above. Hansen, Sargent, and Tallarini (1999) and Hansen and Sargent (2007) introduced the preference for robustness (a concern for model misspecification) into economic models. In robust control problems, agents are concerned about the possibility that their true model is misspecified in a manner that is difficult to detect statistically; consequently, they choose their decisions as if the subjective distribution over shocks was chosen by an evil agent in order to minimize their utility (that is, the solution to a robust decision-maker's problem is the equilibrium of a max-min game between the decisionmaker and the evil agent). ${ }^{7}$ In other words, the objective of robust control is to design a policy that can still work well even if the approximating model (i.e., the best estimate of the true model) does not coincide with the true model. It is worth noting that the key difference between the $\mathrm{RB}$ agent and the Bayesian agent is that the Bayesian agent can form a weight on each model by combining his priors (over the probability of each model being correctly specified) with the data, whereas the RB agent does not have the ability to express his beliefs about the models in terms of well-defined probability distributions. ${ }^{8}$ In the TSH setting, specifically, the preference for RB interacts with the fundamental uncertainty (the government spending and output shocks in the TSH model) and gives rise to a type of induced uncertainty: model uncertainty. ${ }^{9}$ This type of induced uncertainty can affect the model's dynamics even within the linear-quadratic (LQ) framework. ${ }^{10}$ We adopt the LQ-TSH setting in this paper because the main purpose of

not consistent with this test; they also explicitly reject the TSH for Sri Lankan data. Relatedly, Cashin, Olekalns, and Sahay (1998) do not reject tax-smoothing by the central Indian government but do for regional governments.

${ }^{7}$ Note that the assumption that the government has concerns about having the wrong model for its spending or taxation policies is not unusual. For instance, U.S. government spending can be affected by economic conditions and political pressure that is difficult to model and predict.

${ }^{8}$ See Adam (2004) for a discussion on comparing robust and Bayesian decision-making.

${ }^{9}$ As shown in Luo and Young $(2010,2014)$, consumption models can still produce precautionary savings but remain within the class of LQ-Gaussian models, which leads to analytical simplicity.

${ }^{10}$ Note that in the traditional linear-quadratic, linearized, or log-linearized models, uncertainty measured by the variance of the fundamental shock does not affect the model dynamics. 
this paper is to inspect the mechanisms through which the induced uncertainty affects the joint dynamics of government deficits, tax rates, and spending, and it is much more difficult to study this informational friction in non-LQ frameworks. ${ }^{11}$ As argued in Hansen and Sargent (2007), the government's commitment technology is irrelevant under RB if the state transition equation is backward-looking. Therefore, we do not specify the commitment technology of the government in the TSH model of this paper. ${ }^{12}$ After solving the model explicitly, we then examine how model uncertainty due to RB can improve the model's predictions on the important dimensions of the joint dynamics of government deficits, tax rates, and spending we discussed above.

We show that the RB model has the potential to generate the observed joint behavior of government deficits, tax rates, and spending in the U.S., depending on the parameter governing the concern for model misspecification. Specifically, we find that incorporating RB enables the model to better match the data along the two key dimensions: generating higher relative volatility of changes in tax rates to government spending and lower contemporaneous correlation between government deficits and government spending. The main mechanisms behind these improvements rely on the effects of $\mathrm{RB}$ on the marginal propensity to taxation based on permanent government spending and the persistence of the tax and government deficit dynamics, with the key driving force being the interactions between RB and fundamental shocks. Intuitively, as the government bears in mind the possible differences between the model it uses (called the approximating model) and the true model, it is induced to set lower tax rates (in order to minimize distortions) and make the government more sensitive to expected changes in spending. After calibrating the RB parameter using the detection error probability, as advocated by Hansen and Sargent (2007) and used in Luo, Nie, and Young $(2012,2014)$, we find that RB can quantitatively help generate the empirical stochastic properties of the joint dynamics of government deficits, tax rates, and spending.

In addition to improving the basic model's ability to match the unconditional moments of fiscal policy, we are also able to show that the RB model improves the match of conditional moments; specifically, the RB model can pass the bivariate VAR test used in Huang and Lin (1993), Ghosh (1995), Adler (2006), and others, provided the desire for robustness is strong enough. We discipline this desire using detection error probabilities; the result is that the model passes the VAR test using U.S. data from 1960 - 2007.

Our benchmark model assumes that there is only a single shock to government spending.

\footnotetext{
${ }^{11}$ As argued in Hansen and Sargent (2007), if the objective function is not quadratic or the state transition equation is not linear, worst possible distributions due to RB are generally non-Gaussian, which significantly complicates the computational task. See Hansen and Sargent (2007) for detailed discussions on the difficulties in solving the non-LQ RB models, and Bidder and Smith (2012) and Young (2012) for nonlinear approaches that characterize the worst-case distributions.

${ }^{12}$ However, in the literature on robust policy, it may be necessary to model commitment technology if the state transition equation involves expectations. See Kasa (2002) for a discussion on robust policies under commitment and Giordani and Soderlind (2004) for a discussion on discretion and simple rules under RB.
} 
In several extensions of the model, we allow for multiple shocks in the government spending process and a shock to the growth of real GDP; when introducing multiple shocks to the government spending process, we consider two cases: (i) the complete-information case in which the government can distinguish multiple components in the spending process and (ii) the incompleteinformation case in which the government cannot distinguish the multiple components. In these extensions, we show that the full-information RE model still cannot explain the two key unconditional moments and the effects of RB on these moments are similar to those from the benchmark. Applying the VAR tests to these extensions is complicated by the the large standard errors on the decomposition between permanent and transitory government innovations; since the confidence intervals include the benchmark case of no transitory shocks, we are confident in asserting that the VAR test would also be satisfied.

Our paper is related to the recent literature on optimal fiscal policy under model uncertainty, e.g., Karantounias (2013) and Svec (2012). Karantounias (2013) examined optimal fiscal policy in the setting of Lucas and Stokey (1983) in which the representative consumer distrusts the approximating model, and Svec (2012) studied how different types of government conduct optimal fiscal policy when the consumer faces model uncertainty. Like Karantounias (2013), we assume that the model uncertainty is incorporated in the government decision process. Unlike both mentioned papers, the focus of this paper is not to study the optimal fiscal policy; our focus in this paper is to explore how model uncertainty due to $\mathrm{RB}$ helps reconcile both unconditional and conditional joint behavior of government deficits, tax rates, and spending observed in the U.S. economy. ${ }^{13}$

The remainder of the paper is organized as follows. Section 2 reviews a standard FI-RE taxation smoothing model proposed by Barro (1979). Section 3 introduces robustness into the standard TSH model and examines the implications of model uncertainty due to RB for the joint dynamics of optimal taxation and government debt. Section 4 discusses how to use detection error probabilities to calibrate the robustness parameter and presents the calibration results. Section 5 studies the effects of RB in the extensions with multiple shocks. Section 6 concludes.

\section{The Model}

In this section, we propose a full-information rational expectations (FI-RE) version of Barro (1979)'s tax-smoothing model and explore the model's implications for optimal taxation and government debt. We assume that the only asset is a riskfree bond. In choosing the optimal tax rate, the government has perfect information about the model's specification.

\footnotetext{
${ }^{13}$ There is a vast literature on optimal monetary policy under robustness. See Giannoni (2002), Onatski and Williams (2003), Giordani and Soderlind (2004), Cogley et al. (2007), Svensson and Williams (2007), Dennis (2010), Ellison and Sargent (2010), and Woodford (2010).
} 


\subsection{Taxation Smoothing Hypothesis Problem}

In this subsection we present optimal paths of the tax rate and the budget surplus when the government chooses to minimize the present value of excess burden from taxation given the stochastic processes of government spending and real GDP. In the absence of a first-best system of lump-sum taxes, the government seeks to minimize the welfare losses arising from its choice of tax rates. These losses are assumed to be an increasing, convex, and time invariant function of the average tax rate. Specifically, following Barro (1979), Roubini (1988), Bohn (1990), Ghosh (1995), and Lloyd-Ellis, Zhan, and Zhu (2005), we assume that the excess burden of taxation is summarized by a quadratic loss function $c\left(\tau_{t}\right)=\frac{1}{2}\left(\tau_{t}+\varphi\right)^{2}$, which measures the value of real income "wasted" when taxes are $\tau_{t} .{ }^{14}$ The optimization problem of the government can thus be written as

$$
\max _{\left\{\tau_{t}, b_{t+1}\right\}} E_{0}\left[-\frac{1}{2} \sum_{t=0}^{\infty} \beta^{t}\left(\tau_{t}+\varphi\right)^{2}\right],
$$

subject to its flow budget constraint

$$
B_{t+1}=R B_{t}+G_{t}-\tau_{t} Y_{t}
$$

where $E_{0}[\cdot]$ is the government's expectation conditional on its information set at time $0, \beta$ is the government's subjective discount factor, $\tau_{t}$ is the tax rate, $B_{t}$ is the amount of government debt, $G_{t}$ is government spending, $Y_{t}$ is real GDP, and $R \geq 1$ is the gross interest rate.

Suppose that real GDP is growing at a constant rate, $n$. Then (2) can be rewritten as

$$
(1+n) b_{t+1}=R b_{t}+g_{t}-\tau_{t}
$$

where lowercase letters denote corresponding variables expressed as a fraction of real GDP, $Y_{t}$. Solving the government's optimization problem (1) subject to (3) and a no-Ponzi condition yields the following optimal path for the tax rate:

$$
\tau_{t}=\left(R-\frac{(1+n)^{2}}{\beta R}\right) E_{t}\left[b_{t}+\frac{1}{R} \sum_{j=0}^{\infty}\left(\frac{1+n}{R}\right)^{j} g_{t+j}\right],
$$

which is just the permanent government expenditures theory proposed by Barro (1979). ${ }^{15}$ Defining $\widetilde{R}=R /(1+n)$ as the effective interest rate faced by the government, (4) can be written as

$$
\tau_{t}=(1+n)\left(\widetilde{R}-\frac{1}{\beta \widetilde{R}}\right) h_{t}
$$

\footnotetext{
${ }^{14}$ Following Barro (1979), Sargent (1987), Bohn (1998), and Huang and Lin (1993), the value of the parameter, $\varphi$, in the loss function is set such that the loss function has the standard properties of $c^{\prime}(\tau)>0$ and $c^{\prime \prime}(\tau)>0$. Note that the value of $\varphi$ does not affect the stochastic proprties of the joint behavior of the three key fiscal variables.

${ }^{15}$ See Appendix 7.1 for the derivation.
} 
where

$$
h_{t}=E_{t}\left[b_{t}+\frac{1}{(1+n) \widetilde{R}} \sum_{j=0}^{\infty}\left(\frac{1}{\widetilde{R}}\right)^{j} g_{t+j}\right]
$$

is permanent government spending, equal to the sum of government debt and the present discounted value of expected government expenditure. This expression is analogous to permanent income in a standard consumption-savings problem.

Define $\gamma \equiv \frac{1-1 /\left(\beta \widetilde{R}^{2}\right)}{1-1 / \widetilde{R}}$. It is clear that $\gamma \lessgtr 1$ iff $\beta \widetilde{R} \lessgtr 1$. In the literature, the case in which $\beta \widetilde{R}<1$ (i.e., $\gamma<1$ ) is called tax tilting. ${ }^{16}$ In the case in which $n=0$ and $\beta R=1, \gamma=1$. Tax smoothing and tax tilting are two main motivations for a government to run budget deficits. Note that even if government spending (as a fraction of real GDP) is constant over business cycles such that there is no need for tax-smoothing, budget deficits may still arise due to the tax-tilting motivation to shift (tilt) taxes across time. Specifically, if $\beta \widetilde{R}<1$, i.e., the subjective discount rate of the government is high, the government will choose to have low tax rates early and increase tax rates over time to finance its accumulated debt. By contrast, if $\beta \widetilde{R}>1$, the government will choose to have relatively high tax rates early and then use accumulated assets (i.e., negative debts) to reduce tax rates over time. Finally, if $\beta \widetilde{R}=1$, then there is no tax-tilting motive for budget deficits but there would be a strong tax-smoothing motive. Following the literature, we will focus only on the tax-smoothing motive (i.e., the $\beta \widetilde{R}=1$ case) when we examine the stochastic properties of the joint behavior of government taxes, deficits, and spending. In this case, the tax function can be written as

$$
\tau_{t}=(1+n)(\widetilde{R}-1) h_{t}
$$

Note that if $n=0, \tau_{t}=(R-1) h_{t}$ and $h_{t}=E_{t}\left[b_{t}+\sum_{j=0}^{\infty} R^{-j-1} g_{t+j}\right]$.

As shown in Luo and Young $(2010,2014)$ and Luo, Nie, and Young (2012), in order to facilitate the introduction of robustness we reduce the above multivariate model with a general exogenous process to a univariate model with iid innovations that can be solved in closed-form. Specifically, in the above TSH model, if $h_{t}$ is defined as a new state variable, combining (5) with the original government budget constraint, we obtain the following new budget constraint:

$$
h_{t+1}=\widetilde{R} h_{t}-\frac{1}{1+n} \tau_{t}+\frac{1}{1+n} \zeta_{t+1}
$$

where

$$
\zeta_{t+1}=\sum_{j=0}^{\infty}\left(\frac{1}{\widetilde{R}}\right)^{j+1}\left(E_{t+1}-E_{t}\right)\left[g_{t+1+j}\right]
$$

is the time $(t+1)$ innovation to permanent spending. We can thus reformulate the above model as

$$
v\left(h_{0}\right)=\max _{\left\{\tau_{t}\right\}_{t=0}^{\infty}}\left\{E_{0}\left[-\sum_{t=0}^{\infty} \beta^{t} c\left(\tau_{t}\right)\right]\right\}
$$

\footnotetext{
${ }^{16}$ See Ghosh (1995) for a discussion on this topic.
} 
subject to $(7)$, and $v\left(h_{0}\right)$ is the government's value function under FI-RE. ${ }^{17}$

To close the model, we assume that government expenditures evolve according to the following $\operatorname{AR}(1)$ process:

$$
g_{t+1}=\left(1-\rho_{g}\right) \bar{g}+\rho_{g} g_{t}+\epsilon_{t+1},
$$

where $\rho_{g} \in[0,1]$ and $\epsilon_{t+1}$ is an iid shock with mean zero and variance $\omega_{\epsilon}^{2}$. Substituting (10) into (5) and (8) yields:

$$
\begin{aligned}
h_{t} & =b_{t}+\frac{1}{(1+n)\left(\widetilde{R}-\rho_{g}\right)} g_{t}+\frac{\left(1-\rho_{g}\right) \bar{g}}{(1+n)\left(\widetilde{R}-\rho_{g}\right)(\widetilde{R}-1)}, \\
\zeta_{t} & =\frac{1}{\widetilde{R}-\rho_{g}} \epsilon_{t} .
\end{aligned}
$$

Therefore, under the FI-RE hypothesis, the change in optimal taxation can be written as

$$
\Delta \tau_{t}=\frac{\widetilde{R}-1}{\widetilde{R}}\left(E_{t}-E_{t-1}\right)\left[\sum_{j=0}^{\infty}\left(\frac{1}{\widetilde{R}}\right)^{j} g_{t+j}\right]=\frac{\widetilde{R}-1}{\widetilde{R}-\rho_{g}} \epsilon_{t}
$$

which relates the innovation to taxation to government spending shocks. In other words, the optimal tax rate follows a random walk in the standard rational expectations model. Although the optimal plan here is to smooth tax rates so that $\tau_{t}=E_{t}\left[\tau_{t+1}\right]$, it does not mean that $\tau_{t}$ is unaffected by the government spending shock; rather, the optimal tax behavior is to make any changes in taxes unpredictable.

The government budget deficit can be written as

$$
\begin{aligned}
d e f_{t} & \equiv b_{t+1}-b_{t}=(\widetilde{R}-1) b_{t}+\frac{1}{1+n} g_{t}-\frac{1}{1+n} \tau_{t} \\
& =-\sum_{j=1}^{\infty} \widetilde{R}^{-j} E_{t}\left[\Delta g_{t+j}\right]
\end{aligned}
$$

which means the government sets the budget deficit to be equal to expected changes in government spending. Using (10), we obtain $d e f_{t}=\frac{1}{1+n} \frac{1-\rho_{g}}{R-\rho_{g}}\left(g_{t}-\bar{g}\right)$, that is, the government deficit is positively correlated with government spending. We now consider two special cases: (i) when the government spending shock is permanent, i.e., $\rho_{g}=1$ (e.g., expenditures on education and health care), and (ii) when the shock is purely transitory, i.e., $\rho_{g}=0$ (e.g., expenditures on unemployment benefit and natural disaster). From (11) and (12), it is clear that for the first case, it is optimal to absorb a permanent expenditure shock entirely by taxes because

$$
\Delta \tau_{t}=\epsilon_{t} \text { and } d e f_{t}=0 .
$$

\footnotetext{
${ }^{17}$ In the next section, we will introduce robustness directly into this "reduced" TSH model; in Appendix 7.2 we show that the general robust multivariate version delivers the same decision rules because debt evolves deterministically, so that the evil nature cannot distort its law of motion.
} 
In contrast, for the second case,

$$
\Delta \tau_{t}=\frac{\widetilde{R}-1}{\widetilde{R}} \epsilon_{t} \text { and } \operatorname{de} f_{t}=\frac{1}{1+n} \frac{1}{\widetilde{R}} \epsilon_{t}
$$

which means that for a positive government spending shock, $\tau_{t}$ must increase by $\frac{\widetilde{R}-1}{\widetilde{R}} \epsilon_{t}$ and the debt must be raised by $\frac{1}{1+n} \frac{1}{\widetilde{R}} \epsilon_{t}$ to guarantee that the GBC is satisfied. In other words, a proportion of the positive shock, $\frac{\widetilde{R}-1}{\widetilde{R}}$, is absorbed by taxation and the rest $\frac{1}{\widetilde{R}}$ is absorbed by debt. Since $\widetilde{R}$ is close to 1 , most of the shock is absorbed by debt.

\subsection{Model Predictions}

Using (11), it is straightforward to compute the relative volatility of the change in tax rates to government spending, which we denote by $\mu$ :

$$
\mu \equiv \frac{\operatorname{sd}\left(\Delta \tau_{t}\right)}{\operatorname{sd}\left(\Delta g_{t}\right)}=\frac{\widetilde{R}-1}{\widetilde{R}-\rho_{g}} \sqrt{\frac{1+\rho_{g}}{2}},
$$

where sd denotes standard deviation and we use the fact that $\Delta g_{t}=\frac{\left(\rho_{g}-1\right) \epsilon_{t-1}}{1-\rho_{g} L}+\epsilon_{t}$, where $L$ is the lag operator. Using the estimated $\rho_{g}$ reported in Table 1 (linear filter) and assuming that $\widetilde{R}=1.01$, the FI-RE model predicts that $\mu=0.056$. However, in the data as reported in Table 1 , $\mu=1.18$, so the model is not even in the ballpark. ${ }^{18}$ Given the estimated processes of government spending, the model predicts that changes in tax rates (relative to changes in spending) should be much smaller than they are in the data.

Using (12), the model predicts that the contemporaneous correlation between government debt and government spending, corr $\left(d e f_{t}, g_{t}\right)$, is 1 . This model prediction contradicts the empirical evidence: in the data the empirical correlation between them is well below 1 . As reported in Table 1, corr $\left(d e f_{t}, g_{t}\right)=0.73$ (for linear filter) and 0.56 (for HP filter).

In the literature, there are two other common tests on the time series properties of average tax rates and deficits. The first is to test whether tax rates follow a random walk. For example, Barro (1981) found that the average tax rate in the U.S. between 1884 and 1979 follows a random walk. ${ }^{19}$ However, as is well known, this test is not conclusive for the tax-smoothing hypothesis (TSH) because tax rates could also follow a random walk if the rates are determined by a random political process. The second is to test whether the budget balance is informative about future changes in government spending using the VAR techniques. For example, Huang and Lin (1993) and Ghosh (1995) applied Campbell's (1987) VAR approach and found that increases in the budget surplus

\footnotetext{
${ }^{18}$ Given the estimated $\rho_{g}$ using HP filter reported in Table 1 , the RE model predicts that $\mu=0.018$, which is even smaller relative to the data.

${ }^{19}$ Other studies include Trehan and Walsh (1988) for the U.S., Ghosh (1995) for the U.S. and Canada, and Strazicich (1997) for the sub-national governments of Canada and the U.S..
} 
signal future increases in government expenditure, which supports the tax-smoothing hypothesis. ${ }^{20}$ This VAR approach is to formulate the TSH as an expression for budget surplus (or deficits) because it takes into account the entire structure of the TSH model and then use a bivariate VAR for government spending and the budget surplus to evaluate the restrictions imposed by the TSH model. The basic idea of this approach is that using the bivariate VAR, it is possible to compute the predicted time path of the budget deficits that is optimal for the government under the TSH and then compare this optimal path to the actual time path of the budget deficits. Specifically, we compare the optimal path of deficits, de $f_{t}=-\sum_{j=1}^{\infty} \widetilde{R}^{-j} E_{t}\left[\Delta g_{t+j}\right]$, to the actual one, de $f_{t}=(R-1) b_{t}+g_{t}-\tau_{t}$, using the following bivariate VAR: ${ }^{21}$

$$
\left[\begin{array}{c}
\Delta g_{t+1} \\
d e f_{t+1}
\end{array}\right]=\left[\begin{array}{ll}
a_{11} & a_{12} \\
a_{21} & a_{22}
\end{array}\right]\left[\begin{array}{c}
\Delta g_{t} \\
d e f_{t}
\end{array}\right]+\left[\begin{array}{l}
e_{1, t+1} \\
e_{2, t+1}
\end{array}\right],
$$

or $z_{t+1}=A z_{t}+e_{t+1}$, where $e_{1, t+1}$ and $e_{2, t+1}$ are the VAR innovations. Denote $e_{t+1}=\left[\begin{array}{ll}e_{1, t+1} & e_{2, t+1}\end{array}\right]$ and assume that $\Sigma_{e}=E\left[e_{t+1} e_{t+1}^{\prime}\right]$ is the $2 \times 2$ variance-covariance matrix of the reduced-form VAR innovations. ${ }^{22}$ The $k$-step ahead forecast from the VAR is

$$
E_{t}\left[z_{t+k}\right]=A^{k} z_{t}
$$

and (12), def $f_{t}=-\sum_{j=1}^{\infty} \widetilde{R}^{-j} E_{t}\left[\Delta g_{t+j}\right]$, can be rewritten as

$$
\left[\begin{array}{ll}
0 & 1
\end{array}\right] z_{t}=\sum_{j=1}^{\infty} \widetilde{R}^{-j} A^{j}\left[\begin{array}{ll}
1 & 0
\end{array}\right] z_{t} .
$$

Given that both $\Delta g_{t}$ and $d e f_{t}$ are stationary, the infinite sum in the above equation converges to

$$
d e f_{t}^{*}=-\left[\begin{array}{ll}
1 & 0
\end{array}\right] A \widetilde{R}^{-1}\left(I-A \widetilde{R}^{-1}\right)^{-1} z_{t} \equiv \Lambda z_{t}=\lambda_{1} \Delta g_{t}+\lambda_{2} d e f_{t}
$$

Therefore, if the null hypothesis that the government smooths taxes over time is correct, the recovered coefficients from the estimated VAR, $\lambda_{1}$ and $\lambda_{2}$, should be 0 and 1 , respectively. We will use this test in Section 4; to satisfy the reader's curiosity for the moment, we note here that a joint test of these restrictions under rational expectations has a Wald statistic of 24.9 on data from $1960-2007$, which is about as strong a rejection as one is likely to see (see Table 6).

\footnotetext{
${ }^{20}$ Adler (2006) tested the TSH using Swedish data from the period 1952-1999, and found that the tax smoothing behavior of the government can explain a significant fraction of the variability in the Swedish government budget deficits.

${ }^{21}$ As argued in Campbell (1987), Huang and Lin (1993), Ghosh (1995), and others, although the information set of an econometrician is only a subset of the government's information set, the econometrician can still compute the predicted path of the budget deficit because the deficit itself contains all of the information about future changes in government spending. That is, the budget deficit should Granger-cause changes in government spending.

${ }^{22}$ See Appendix 7.3 for deriving the standard errors for the VAR test.
} 


\section{Incorporating Robustness}

\subsection{The RB Version of the Tax Smoothing Hypothesis Model}

Robust control emerged in the engineering literature in the 1970s and was introduced into economics and further developed by Hansen, Sargent, and others. A simple version of robust optimal control considers the question of how to make decisions when the agent does not know the probability model that generates the data. Specifically, an agent with a preference for robustness considers a range of models surrounding the given approximating model, (7):

$$
h_{t+1}=\widetilde{R} h_{t}-\frac{1}{1+n} \tau_{t}+\widetilde{\zeta}_{t+1}+\omega_{\widetilde{\zeta}} \nu_{t}
$$

where $\widetilde{\zeta}_{t+1}=\frac{1}{1+n} \zeta_{t+1}$ and $\omega_{\widetilde{\zeta}}=\frac{1}{1+n} \omega_{\zeta}$, and makes decisions that maximize lifetime expected utility given this worst possible model (i.e., the distorted model). To make that model (7) is a good approximation when (15) generates the data, we constrain the approximation errors by an upper bound $\eta_{0}$ :

$$
E_{0}\left[\sum_{t=0}^{\infty} \beta^{t+1} \nu_{t}^{2}\right] \leq \eta_{0},
$$

where $E_{0}[\cdot]$ denotes conditional expectations evaluated with the model, and the left side of this inequality is a statistical measure of the discrepancy between the distorted and approximating models. Note that the standard full-information RE case corresponds to $\eta_{0}=0$. In the general case in which $\eta_{0}>0$, the evil agent is given an intertemporal entropy budget $\eta_{0}>0$ which defines the set of models that the agent is considering. Following Hansen and Sargent (2007), we compute robust decision rules by solving the following two-player zero-sum game: a minimizing decision maker chooses optimal taxation $\left\{\tau_{t}\right\}$ and a maximizing evil agent chooses model distortions $\left\{\nu_{t}\right\}$. Specifically, the robustness version of the Barro model proposed in Section 2 can be written as

$$
v\left(h_{t}\right)=\max _{\tau_{t}} \min _{\nu_{t}}\left\{-\frac{1}{2}\left(\tau_{t}+\varphi\right)^{2}+\beta\left[\vartheta \nu_{t}^{2}+E_{t}\left[v\left(h_{t+1}\right)\right]\right]\right\}
$$

subject to the distorted transition equation (i.e., the worst-case model), (15), where $\nu_{t}$ distorts the mean of the innovation, and $\vartheta>0$ is the Lagrange multiplier on the constraint specified in (16) and controls how bad the error can be. ${ }^{23}$

When the ratio of government spending to real GDP follows the AR(1) process, (10), solving this robust control problem yields the following proposition:

\footnotetext{
${ }^{23}$ Formally, this setup is a game between the decision-maker and a malevolent nature that chooses the distortion process $\nu_{t} . \vartheta \geq 0$ is a penalty parameter that restricts attention to a limited class of distortion processes; it can be mapped into an entropy condition that implies agents choose rules that are robust against processes which are close to the trusted one. In a later section we will apply an error detection approach to calibrate $\vartheta$ in the U.S. economy.
} 
Proposition 1 Under RB, the optimal tax function is

$$
\tau_{t}=\frac{(1+n)(\widetilde{R}-1)}{1-\Sigma} h_{t}+\frac{\Sigma \varphi}{1-\Sigma},
$$

the mean of the worst-case shock is

$$
\nu_{t} \omega_{\widetilde{\zeta}}=\frac{(\widetilde{R}-1) \Sigma}{1-\Sigma} h_{t}+\frac{1}{1+n} \frac{\Sigma \varphi}{1-\Sigma},
$$

the government deficit can be written as

$$
d e f_{t}=b_{t+1}-b_{t}=\frac{1}{1+n} \frac{1-\rho_{g}}{\widetilde{R}-\rho_{g}}\left(g_{t}-\bar{g}\right)-\left(1-\rho_{h}\right) h_{t}-\frac{1}{1+n} \frac{\Sigma \varphi}{1-\Sigma},
$$

and $h_{t}\left(=b_{t}+\frac{1}{(1+n)\left(\widetilde{R}-\rho_{g}\right)} g_{t}+\frac{\left(1-\rho_{g}\right) \bar{g}}{(1+n)\left(\widetilde{R}-\rho_{g}\right)(\widetilde{R}-1)}\right)$ is governed by:

$$
h_{t+1}=\rho_{h} h_{t}-\frac{1}{1+n} \frac{\Sigma \varphi}{1-\Sigma}+\widetilde{\zeta}_{t+1}
$$

in the approximating model, where $\zeta_{t+1}=\frac{1}{\widetilde{R}-\rho_{g}} \epsilon_{t+1}, \widetilde{\zeta}_{t+1}=\frac{1}{1+n} \zeta_{t+1}$,

$$
\Sigma=(1+n)^{2} \widetilde{R} \omega_{\widetilde{\zeta}}^{2} /(2 \vartheta)=\widetilde{R} \omega_{\zeta}^{2} /(2 \vartheta)>0
$$

measures the degree of the preference of robustness, and $\rho_{h}=\frac{1-\widetilde{R} \Sigma}{1-\Sigma} \in(0,1)$.

Proof. See Appendix 7.4.

The univariate $\mathrm{RB}$ model with unique state variable $h$ leads to the same taxation function as the corresponding multivariate RB model in which the state variables are $b_{t}$ and $g_{t}$. The key difference between these two models is that in the univariate RB model the evil agent distorts the transition equation of permanent spending $h_{t}$, whereas in the multivariate RB model the evil agent distorts the spending-output ratio process $g_{t}$. Theoretically, introducing RB into the multivariate model affects both the coefficients attached to $b_{t}$ and $g_{t}$ in the taxation function. That is, in the multivariate model RB may affect the relative importance of the two state variables in the taxation function, whereas in the univariate model the relative importance of the two effects are fixed by reducing the $(b, g)$ state space to the univariate state $h$. However, after solving the twostate model numerically using the standard procedure proposed in Hansen and Sargent (2007), we can see that the two models lead to the same decision rule (see Appendix 7.2); the intuition is that the debt evolves deterministically from $t$ to $t+1$, so that the evil agent cannot influence it. The main reason that we adopt the univariate $\mathrm{RB}$ model here is to obtain the explicit expressions for optimal taxation and government debts which can be easily used to compute the model's stochastic properties (e.g., the relative volatility of taxation and debts to government spending 
and the correlation between government debts and spending) and form the VAR test conducted in Section 4.4 .

The effect of the preference for robustness, $\Sigma$, is jointly determined by the RB parameter, $\vartheta$, and the volatility of the permanent spending shock, $\omega_{\widetilde{\zeta}}$. This interaction provides a novel channel through which the government spending shock affects optimal taxation and debts. Specifically, optimal taxation under RB, (18), shows that the preference for robustness, $\vartheta$, affects the optimal response of optimal taxation to the change in permanent government expenditures, $(1+n) \frac{\widetilde{R}-1}{1-\Sigma}$. The smaller the value of $\vartheta$ the larger the response of optimal taxation; that is, under RB optimal taxation is more sensitive to unanticipated government spending shocks. This response is referred to as "making hay while the sun shines" in van der Ploeg (1993) to explain the overreaction of consumption to unanticipated income shocks, and reflects the precautionary aspect of these preferences.

\section{Proposition $2 \Sigma<1$.}

Proof. The second-order condition for a minimization by nature is

$$
A=\frac{1}{2} \frac{(1+n)^{2} \widetilde{R}(\widetilde{R}-1)}{1-(1+n)^{2} \widetilde{R} \omega_{\widetilde{\zeta}}^{2} /(2 \vartheta)}>0
$$

which can be rearranged into

$$
\vartheta>(1+n)^{2} \widetilde{R} \omega_{\widetilde{\zeta}}^{2}
$$

Using the definition of $\Sigma$ we obtain

$$
1>\Sigma \text {. }
$$

It is straightforward to show that the robust policy rule, (18), can also be obtained by solving the following risk-sensitive TSH model:

$$
v\left(h_{t}\right)=\max _{\tau_{t}}\left\{-\frac{1}{2}\left(\tau_{t}+\varphi\right)^{2}+\beta \mathcal{R}_{t}\left[v\left(h_{t+1}\right)\right]\right\}
$$

subject to (7), and the distorted expectation operator $\mathcal{R}_{t}$ is defined by

$$
\mathcal{R}_{t}\left[v\left(h_{t+1}\right)\right]=-\frac{1}{\alpha} \log E_{t}\left[\exp \left(-\alpha v\left(h_{t+1}\right)\right)\right],
$$

where $\alpha>0$ measures higher risk aversion of the government vis a vis the von NeumannMorgenstern specification. ${ }^{24}$ Risk-sensitivity (RS) was first introduced into the LQG framework

\footnotetext{
${ }^{24}$ The detailed proof is available from the authors by request. The observational equivalence between the risksensitive and robust LQG models has been well established in the literature. See Hansen, Sargent, and Tallarini (1999), Backus, Routledge, and Zin (2004), and Luo and Young (2010).
} 
by Jacobson (1973) and extended by Whittle (1981). van der Ploeg (1993) applied this preference to examine its implication for precautionary savings; Hansen and Sargent (1995) introduced discounting into the RS specification and showed that the resulting decision rules are time-invariant; Hansen, Sargent, and Tallarini (1999) also explored its implications for precautionary savings and asset prices; and Luo and Young (2010, 2014) examined its implications for consumption and precautionary savings when consumers are inattentive. In the risk-sensitive TSH specified in (23), the government is prudent in the sense that they minimize the expected value of an exponential transformation of a quadratic welfare loss function and adjust optimal taxation policy more aggressively to changes in government expenditures. Note that as examined in Hansen and Sargent (2007), we can obtain the same Bellman equation as (23) if using the multiplier preference to model RB:

$$
v\left(h_{t}\right)=\max _{\tau_{t}}\left\{-\frac{1}{2}\left(\tau_{t}+\varphi\right)^{2}+\beta \min _{m_{t+1}}\left\{E_{t}\left[m_{t+1} v\left(h_{t+1}\right)\right]+\vartheta E_{t}\left[m_{t+1} \ln m_{t+1}\right]\right\}\right\},
$$

where $E_{t}\left[m_{t+1} \ln m_{t+1}\right]$ is the relative entropy of the distribution of the distorted model with respect to that of the approximating model, and $\vartheta>0$ is the shadow price of capacity that can reduce the distance between the two distributions, i.e., the Lagrange multiplier on the constraint: $E_{t}\left[m_{t+1} \ln m_{t+1}\right] \leq \eta$. Minimizing with respect to $m_{t+1}$ yields $m_{t+1}=\frac{\exp \left(-v\left(h_{t+1}\right) / \vartheta\right)}{E_{t}\left[\exp \left(-v\left(h_{t+1}\right) / \vartheta\right)\right]}$, and it is straightforward to show that substituting $m_{t+1}$ into (24) yields (23).

\subsection{Implications of RB for Tax Smoothing and Government Debt}

Given the expression for optimal taxation (18), the dynamics of taxes can be written as

$\tau_{t+1}=\rho_{h} \tau_{t}+\frac{(1+n)(\widetilde{R}-1)}{1-\Sigma} \widetilde{\zeta}_{t+1}-(\widetilde{R}-1) \frac{\Sigma \varphi}{1-\Sigma}$, or $\tau_{t+1}=\rho_{h} \tau_{t}+\frac{\widetilde{R}-1}{1-\Sigma} \zeta_{t+1}-(\widetilde{R}-1) \frac{\Sigma \varphi}{1-\Sigma}$,

and the government deficit can be rewritten as the AR(1) process

$$
d e f_{t+1}=\rho_{h} d e f_{t}+\frac{1}{1+n} \frac{\left(1-\rho_{g}\right)\left(\rho_{g}-\rho_{h}\right)}{\widetilde{R}-\rho_{g}}\left(g_{t}-\bar{g}\right)+\left(\rho_{h}-\rho_{g}\right) \widetilde{\zeta}_{t+1} .
$$

Note that even if the government's subjective discount factor is assumed to equal the inverse of the effective interest rate $(\beta \widetilde{R}=1)$, the tax-tilting effect of government budget deficits still operates under RB. The intuition is very simple: the effect of RB on optimal taxation and deficits are the same as that of the discount factor within the LQ setting. ${ }^{25}$ We also note that the tax

\footnotetext{
${ }^{25}$ See Hansen, Sargent, and Tallarini (1999) and Luo and Young (2010) for discussions on the observational equivalence (OE) between RB and the discount factor in the LQ permanent income models. However, it is worth noting that this OE only holds in the "static" sense. For example, if we allow the value of DEP $(p)$ to change, the calibrated value of $\vartheta$, the implied value of $\Sigma$, and the resulting model dynamics will change accordingly. In addition, when the fundamental uncertainty changes, the calibrated $\vartheta$, the implied value of $\Sigma$, and the resulting model dynamics will also change accordingly. In contrast, the discount factor can be very stable or constant over time and across states.
} 
rate is a stationary process even if $g$ is a random walk, in contrast to the FI-RE model.

Using (25) and (26), the following proposition summarizes the main results on the relative volatility of the change in tax rates to government spending and the contemporaneous correlation between government debt and spending:

Proposition 3 Under $R B$, the relative volatility of the change in tax rates to government spending, $\mu$, is:

$$
\mu \equiv \frac{\operatorname{sd}\left(\Delta \tau_{t}\right)}{\operatorname{sd}\left(\Delta g_{t}\right)}=\frac{\widetilde{R}-1}{(1-\Sigma)\left(\widetilde{R}-\rho_{g}\right)} \sqrt{\frac{1+\rho_{g}}{1+\rho_{h}}}
$$

where we use the fact that $\Delta g_{t}=\frac{\left(\rho_{g}-1\right) \epsilon_{t-1}}{1-\rho_{g} L}+\epsilon_{t}$. The contemporaneous correlation between the government debt and government spending, $\operatorname{corr}\left(d e f_{t}, g_{t}\right)$, is:

$$
\operatorname{corr}\left(d e f_{t}, g_{t}\right)=\left(\frac{1}{1+\rho_{g}}-\frac{1-\rho_{h}}{1-\rho_{g} \rho_{h}}\right) /\left[\sqrt{\frac{1}{1-\rho_{g}^{2}}} \sqrt{\frac{1-\rho_{g}}{1+\rho_{g}}+\frac{1-\rho_{h}}{1+\rho_{h}}-2 \frac{\left(1-\rho_{h}\right)\left(1-\rho_{g}\right)}{1-\rho_{g} \rho_{h}}}\right] .
$$

\section{Proof. See Appendix 7.5.}

It is clear from (27) that RB increases the relative volatility via two channels: first, it increases the marginal propensity to tax based on permanent government spending $\left(\frac{\widetilde{R}-1}{1-\Sigma}\right)$; and second, it increases the volatility of the change in the tax rate by reducing the persistence of the tax dynamics. The latter is because the persistence of tax dynamics is the same as the persistence of

permanent government spending measured by $\rho_{h}$ and it is easy to show $\frac{\partial \rho_{h}}{\partial \Sigma}<0$. Figure 2 provides a graphic view how this relative volatility decreases with the degree of model uncertainty, $\Sigma$, for different values of $\rho_{g}$.

From (28), we can see that under RB, the correlation converges to 1 as $\Sigma$ converges to 0 . Figure 3 illustrates how RB affects the correlation between the government debt and government spending for different values of $\rho_{g}$. It is clear that corr $\left(d e f_{t}, g_{t}\right)$ is decreasing with $\Sigma$. If we decompose this correlation into two terms, the covariance between deficit and spending (the nominator) and the variances (the denominator), we find that the decrease of the correlation between deficit and spending is mainly driven by the decrease in the covariance term as the degree of model uncertainty $(\Sigma)$ increases. Using the explicitly derived expression for this covariance term, cov $\left(d e f_{t}, g_{t}\right)$, it can be shown that $\operatorname{cov}\left(d e f_{t}, g_{t}\right)$ is a monotonically increasing function of the persistence of the deficit function, measured by $\rho_{h}$. Thus, as an increase in the degree of model uncertainty $(\Sigma)$ reduces $\rho_{h}$, cov $\left(d e f_{t}, g_{t}\right)$ falls. Therefore, as FI-RE over-predicts the correlation between debt and spending, RB aligns the model and the data more closely along this dimension. 


\subsection{Two Special Cases}

\subsubsection{A Permanent Increase in Government Spending}

Under RB, when $\rho_{g}=1$, we have

$$
\begin{aligned}
\Delta \tau_{t} & =\frac{\rho_{h}-1}{1-\Sigma} \frac{1}{1-\rho_{h} L} \epsilon_{t-1}+\frac{1}{1-\Sigma} \epsilon_{t}, \\
d e f_{t} & =-\frac{1}{1+n} \frac{\Sigma}{1-\Sigma} \frac{1}{1-\rho_{h} L} \epsilon_{t}=-\frac{1}{1+n} \frac{\Sigma}{1-\Sigma} \frac{1}{1-\rho_{h} L} \epsilon_{t-1}-\frac{1}{1+n} \frac{\Sigma}{1-\Sigma} \epsilon_{t} .
\end{aligned}
$$

It is clear that in this permanent income case, a proportion $\frac{1}{1-\Sigma}$ of a newly-arrived shock $\left(\epsilon_{t}\right)$ is absorbed by $\tau_{t}$ and $-\frac{1}{1+n} \frac{\Sigma}{1-\Sigma}$ of the shock is absorbed by debt. In other words, taxation is more sensitive to the permanent shock under RB and thus the government debt can be reduced even if the spending shock is positive. In addition, both taxes and debts react to the lagged innovations to government spending. This prediction is clearly different from what we obtained in the $\mathrm{RE}$ model: the permanent expenditure shock is absorbed entirely by taxes.

\subsubsection{A Temporary Increase in Government Spending}

Under RB, when $\rho_{g}=0$, we have

$$
\begin{aligned}
\Delta \tau_{t} & =\frac{\left(\rho_{h}-1\right)(\widetilde{R}-1)}{(1-\Sigma)} \frac{\epsilon_{t-1}}{1-\rho_{h} L}+\frac{\widetilde{R}-1}{(1-\Sigma) \widetilde{R}} \epsilon_{t}, \\
\operatorname{de} f_{t} & =\frac{1}{1+n}\left[\frac{1}{\widetilde{R}} \epsilon_{t}-\frac{\Sigma(\widetilde{R}-1)}{(1-\Sigma) \widetilde{R}} \frac{\epsilon_{t}}{1-\rho_{h} L}\right]=\frac{1}{1+n}\left[-\frac{\Sigma(\widetilde{R}-1)}{(1-\Sigma) \widetilde{R}} \frac{\rho_{h}}{1-\rho_{h} L} \epsilon_{t-1}+\frac{1-\Sigma \widetilde{R}}{(1-\Sigma) \widetilde{R}} \epsilon_{t}\right],
\end{aligned}
$$

which means that for a positive government spending shock $\left(\epsilon_{t}\right), \tau_{t}$ must increase by $\frac{\widetilde{R}-1}{(1-\Sigma) \widetilde{R}} \epsilon_{t}$ and the debt must be reduced by $\frac{1-\Sigma \widetilde{R}}{(1-\Sigma) \widetilde{R}} \epsilon_{t} \cdot{ }^{26}$ In other words, a proportion of the positive shock, $\frac{\widetilde{R}-1}{(1-\Sigma) \widetilde{R}}$, is absorbed by taxation and the rest, $\frac{1-\Sigma \widetilde{R}}{(1-\Sigma) \widetilde{R}}$, is absorbed by debt. Note that in the RE model a proportion of the shock, $\frac{\widetilde{R}-1}{\widetilde{R}}$, is absorbed by taxation and the rest, $\frac{1}{\widetilde{R}}$, is absorbed by debt. Since $\Sigma>0$, it is clear that RB strengthens the relative importance of taxation to government debts in absorbing the government spending shock.

\section{Calibration and Main Findings}

In the previous section we have seen that the model uncertainty due to the preference for robustness can help better explain the observed relative volatility of the tax rate and the correlation

\footnotetext{
${ }^{26}$ Note that here we need to impose the restriction that $\Sigma<1 / \widetilde{R}$, which is the breakdown condition discussed in Hansen and Sargent (2007); for values of $\Sigma$ that violate this condition, nature can reduce expected utility to $-\infty$.
} 
between government budget deficit and government spending. Specifically, we have shown that an increase of $\Sigma$ (which measures the RB effect) increases the relative volatility of the tax rate and reduces the correlation between government budget deficit and government spending. But careful readers may still have questions on how to further interpret this parameter. For example, two very reasonable questions are: What does $\Sigma$ really measure? How should we link the value of $\Sigma$ to the degree of model uncertainty? In this section, we will provide one useful method to quantify the RB parameter, $\Sigma$, which intuitively links it to the degree of model uncertainty.

\subsection{Calibrating the RB Parameter}

We follow Hansen, Sargent, and Wang (2002) and Hansen and Sargent (2007) to calibrate the RB parameter $(\vartheta$ and $\Sigma$ ). Specifically, we calibrate the model by using the model detection error probability that is based on a statistical theory of model selection (the approach will be precisely defined below). We can then infer what values of the RB parameter $\vartheta$ imply reasonable fears of model misspecification for empirically-plausible approximating models. In other words, the model detection error probability is a measure of how far the distorted model can deviate from the approximating model without being discarded; standard significance levels for testing are then used to determine what reasonable fears entail.

\subsubsection{The Definition of the Model Detection Error Probability}

Let's use model $A$ to denote the approximating model and model $B$ to denote the distorted model. Define $p_{A}$ as

$$
p_{A}=\operatorname{Prob}\left(\log \left(\frac{L_{A}}{L_{B}}\right)<0 \mid A\right),
$$

where $\log \left(\frac{L_{A}}{L_{B}}\right)$ is the log-likelihood ratio. When model $\mathrm{A}$ generates the data, $p_{A}$ measures the probability that a likelihood ratio test selects model $B$. In this case, we call $p_{A}$ the probability of the model detection error. Similarly, when model $B$ generates the data, we can define $p_{B}$ as

$$
p_{B}=\operatorname{Prob}\left(\log \left(\frac{L_{A}}{L_{B}}\right)>0 \mid B\right) .
$$

Following Hansen, Sargent, and Wang (2002) and Hansen and Sargent (2007), we can define the detection error probability, $p$, as the average of $p_{A}$ and $p_{B}$ :

$$
p(\vartheta)=\frac{1}{2}\left(p_{A}+p_{B}\right)
$$

where $\vartheta$ is the robustness parameter used to generate model $B$. By this definition, $1-p$ measures the probability that econometricians can distinguish the approximating model from the distorted model. The next subsection shows how to compute the model detection error probability in the RB model. 


\subsubsection{Calibrating the RB Parameter in the Tax-smoothing Model}

Under RB, assuming that the approximating model generates the data, the state, $h_{t}$, evolves according to the transition law

$$
\begin{aligned}
h_{t+1} & =\widetilde{R} h_{t}-\frac{1}{1+n} \tau_{t}+\widetilde{\zeta}_{t+1}, \\
& =\frac{1-\widetilde{R} \Sigma}{1-\Sigma} h_{t}-\frac{1}{1+n} \frac{\Sigma \varphi}{1-\Sigma}+\widetilde{\zeta}_{t+1} .
\end{aligned}
$$

In contrast, assuming that the distorted model generates the data, $h_{t}$ evolves according to

$$
\begin{aligned}
h_{t+1} & =\widetilde{R} h_{t}-\frac{1}{1+n} \tau_{t}+\widetilde{\zeta}_{t+1}+\omega_{\widetilde{\zeta}} \nu_{t} \\
& =h_{t}+\widetilde{\zeta}_{t+1}
\end{aligned}
$$

Because permanent shocks are both damaging (they imply infinite long-run variances) and difficult to detect in small samples (unit root tests have low power), the government designs its tax policy to be robust against them.

To compute $p_{A}$ and $p_{B}$, we use the following procedure:

1. Simulate $\left\{h_{t}\right\}_{t=0}^{T}$ using (36) and (37) a large number of times. The number of periods used in the simulation, $T$, is set to be the actual length of the U.S. data.

2. Count the number of times that $\log \left(\frac{L_{A}}{L_{B}}\right)<0 \mid A$ and $\log \left(\frac{L_{A}}{L_{B}}\right)>0 \mid B$ are each satisfied.

3. Determine $p_{A}$ and $p_{B}$ as the fractions of realizations for which $\log \left(\frac{L_{A}}{L_{B}}\right)<0 \mid A$ and $\log \left(\frac{L_{A}}{L_{B}}\right)>0 \mid B$, respectively.

In the above simulation, the volatility of $\zeta_{t}$ is computed using $\operatorname{sd}(\zeta)=\frac{\sqrt{1-\rho_{g}^{2}}}{R-\rho_{g}} \operatorname{sd}(g)$, where $\operatorname{sd}(g)$ is the standard deviation of government spending. $\varphi$ is set to be $-\bar{\tau}$ where $\bar{\tau}$ is the average tax rate in the data. ${ }^{27}$ It is worth pointing out that the detection error probability, $p$, declines with time. That is, for a given value of the RB parameter $(\Sigma)$, the value of $p$ calculated in the above simulation declines as the number of simulation periods increases. Figure 4 shows how $p$ varies with the simulation length under three different values of $\Sigma$. The solid line uses the calibrated value of $\Sigma$ (as reported in Table 2). The dot-dashed and dashed lines use values of 0.9 and 0.8 , respectively. The three lines share the same pattern: $p$ declines with the simulation length. The interpretation is that the probability of being unable to distinguish the approximating model and the distorted model declines with more available data.

\footnotetext{
${ }^{27}$ We follow Luo, Nie, and Young (2012) and use the local coefficient of relative risk aversion for the loss function to calibrate $\varphi$.
} 


\subsection{Data}

To implement the calibration described in the previous section, we need to have the measure of GDP, government spending $\left(G_{t}\right)$, government debt $\left(B_{t}\right)$, government budget deficit $\left(d e f_{t}\right)$, tax rate $\left(\tau_{t}\right)$, and risk-free interest rate. We follow Huang and Lin (1993) and Lloyd-Ellis et al. (2005) to construct these variables and use the same data sources as described in their papers. Specifically, government expenditures are constructed by using federal expenditures minus net federal interest payments. The data on both variables are taken from National Income and Product Accounts (NIPA). Government budget deficit is defined as total outlays minus total receipts. ${ }^{28}$ Government debt is defined as the federal debt (end of period) held by the public. Tax rate is defined as tax revenues divided by GDP. The data on these variables are taken from The Economic Report of President, 2011. The data covers the period $1960-2007 .{ }^{29}$ Finally, we need to know the annual growth rate of real GDP, $n$, and the risk-free interest rate, $R$. Using the real GDP data from NIPA, the former is calculated as the average annual growth rate of real GDP in the period of $1960-2007$, which is $3 \%$. We set the risk-free rate to be 1.04 such that the effective interest rate $\widetilde{R}=1.01$.

For variables as a ratio of GDP, we use either a linear filter or the Hodrick-Prescott (HP) filter (with a smoothing parameter of 100) to detrend the data. ${ }^{30}$ For variables expressed in the form of differences (with a symbol $\Delta$ ) the unfiltered series are used.

\subsection{Main Results}

The calibration results are reported in Table 2 . We set $p=0.1$ which means that there exists a 10-percent chance a likelihood ratio test fails to separate the approximating model and the distorted model. The resulting value of the RB parameter $\Sigma$ is 0.96 . In the same table, we also report the persistence of the government spending (as a ratio of GDP), $g$, and the measured coefficient of variation for the processes of $g$ and $\zeta$ (the innovation to the $h_{t}$ process defined in budget constraint equation (7)).

As shown in the previous section, introducing model uncertainty (concern for robustness) qualitatively improves the model performance in explaining the two unconditional moments: (1) the relative standard deviation of the tax rate, $\frac{\operatorname{sd}(\Delta \tau)}{\operatorname{sd}(\Delta g)}$; and (2) the correlation between government budget deficit and government spending, $\operatorname{corr}(d e f, g)$. Specifically, we have shown that an increase of $\Sigma$ can increase the relative standard deviation of the tax rate while reducing the correlation of the government budget deficit and government spending. Since we now have a calibrated value

\footnotetext{
${ }^{28}$ In Lloyd-Ellis, Zhan, and Zhu (2005), the authors use the government budget surplus, which is defined as total receipts minus total outlays.

${ }^{29}$ As US government debt remained at very high levels during and after the Korean War period, here we excludes the high debt years surrounding the Korean War. In addition, we also exclude the 2008-2010 financial crisis period.

${ }^{30}$ We report the values of the key moments using both filters in Table 1.
} 
of $\Sigma$, we can quantitatively show these improvements more clearly by comparing the predicted values from the $\mathrm{RE}$ and the $\mathrm{RB}$ models. The results are reported in Table 3.

The first column of Table 3 lists the values of the two moments in the data. The second column reports predictions of the $\mathrm{RE}$ model with no model uncertainty. As it shows, the relative volatility of the tax rate predicted by the RE model is only 0.04 while it is 1.18 in the data. On the other hand, the RE model overpredicts the correlation between government budget deficit and government spending. As a comparison, the $\mathrm{RB}$ (at $p=0.1$ ) can predict values that are much closer to the data, as shown in the third column. These results confirm our theoretic findings in Section 3. In that section, we have shown that as the RB effect (measured by $\Sigma$ ) increases, the model can predict more reasonable relative volatility of the tax rate and the correlation between deficit and spending. The results in Table 3 present one numerical example of the magnitude of the improvements. Of course, the improvements depend on the detection error probability that is used.

As we have explained before, using the model detection error probability $(p)$ is one intuitive way to measure the model uncertainty. In this example, we set $p=0.1$ so that agents are considering a range of models which cannot be distinguished by a likelihood ratio test up to a probability of $0.1 .^{31}$ Alternatively speaking, this means that agents suspect that the model is misspecified to a certain degree that a likelihood ratio test cannot separate the (unknown) true model with other similar models by a 10-percent chance. To see how the degree of model uncertainty affects the model prediction and provide more robust check, we vary the detection error probability $p$ (or the degree of model uncertainty) and report the corresponding results in Table 5. The corresponding calibrated values of $\Sigma$ are reported in Table 4 . Remember that as the detection error probability $(p)$ increases, it becomes more difficult (using a likelihood-ratio test) to distinguish the set of considered models. Thus the range of models considered have to be smaller. In other words, there is less model uncertainty or agents have less concern about the model misspecifications. As Table 5 shows, as the degree of the model uncertainty decreases, the relative volatility of the tax rate declines and the correlation between deficit and government spending increases. These findings again confirm the theoretic results in the previous sections.

\subsection{Implications of RB for the Bivariate VAR Test on the TSH}

In this subsection, we examine the implications of RB for the bivariate VAR test on the TSH mentioned in Section 2.2. Using the optimal taxation function under RB, $\tau_{t}=\frac{(1+n)(\widetilde{R}-1)}{1-\Sigma} h_{t}+\frac{\Sigma \varphi}{1-\Sigma}$, obtained in Section 3.1., the resulting deficit equation can be written as

$$
d e f_{t}=-\sum_{j=0}^{\infty} \widetilde{R}^{-j} E_{t}\left[\Delta g_{t+j}\right]-\Sigma \tau_{t} \text { or } \widetilde{d e f} f_{t}=-\sum_{j=0}^{\infty} \widetilde{R}^{-j} E_{t}\left[\Delta g_{t+j}\right]
$$

\footnotetext{
${ }^{31}$ A detection probability of 0.1 is what Hansen and Sargent (2007) proposes.
} 
where $\widetilde{d e f} t=d e f_{t}+\Sigma \tau_{t}$. Following the same procedure as discussed in Section 2.2, the VAR can be formulated as

$$
\left[\begin{array}{c}
\Delta g_{t+1} \\
\widetilde{d e f}_{t+1}
\end{array}\right]=\left[\begin{array}{ll}
a_{11} & a_{12} \\
a_{21} & a_{22}
\end{array}\right]\left[\begin{array}{c}
\frac{\Delta g_{t}}{\widehat{d e f}_{t}}
\end{array}\right]+\left[\begin{array}{c}
e_{1, t+1} \\
e_{2, t+1}
\end{array}\right] .
$$

Table 6 reports the recovered $\lambda_{1}$ and $\lambda_{2}$ from the full-information RE and RB models using data for the period from $1960-2007$. To investigate how model uncertainty affects test results, we report nine different $\mathrm{RB}$ models in which $\Sigma$ varies from 0.1 to 0.9 . Remember that a larger $\Sigma$ means more model uncertainty. ${ }^{32}$ The FI-RE model can be considered a model with zero model uncertainty. Thus, in total, Table 6 reports the recovered values for $\lambda_{1}$ and $\lambda_{2}$ for ten models differing only in the degree of model uncertainty, which increases from zero (the FI-RE model) to a significantly large degree (measured by $\Sigma$ ). The second and third columns show that $\lambda_{1}$ decreases with the degree of model uncertainty while $\lambda_{2}$ increases with it. More importantly, the increase of model uncertainty leads $\lambda_{1}$ to decrease from a positive level (although not statistically significant) to a level very close to 0 and $\lambda_{2}$ to rise significantly toward 1 . In other words, RB can help make the model better fit the data because the recovered coefficients from the estimated VAR, $\lambda_{1}$ and $\lambda_{2}$, should be 0 and 1 , respectively, if the TSH holds. ${ }^{33}$ More formally, the last two columns report the Wald statistics and the associated $p$-values for the joint hypothesis of $\lambda_{1}=0$ and $\lambda_{2}=1$. The Wald statistic decreases and the associated $p$-value increases as the degree of model uncertainty increases. Of particular interest is that the values of $\Sigma$ consistent with reasonable detection error probabilities - namely, those above 0.9 - are ones which potentially could pass the VAR test. 34

\section{Extensions: Multiple Shocks}

In this section we consider some extensions to the benchmark, to demonstrate how robust our results on robustness are. Specifically, we consider decomposing government spending into a permanent and a transitory component; we study cases where the government can and cannot distinguish between the two shocks. Finally, we investigate the addition of shocks to the growth rate of total output. Our results survive each of these extensions.

\footnotetext{
${ }^{32}$ As explained in the previous section, a larger $\Sigma$ corresponds to a smaller the detection error probability which means that the difference between the distorted model and the approximating model is larger. In other words, the range of the models (around the approximating model) is larger, or, there is more model uncertainty faced by the decision maker.

${ }^{33} \mathrm{An}$ alternative interpretation is that incorporating certain degree of model uncertainty makes the TSH be less likely to be rejected in the data.

${ }^{34}$ The breakdown condition occurs at $\Sigma=0.9901$, so we could in principle increase the value of $\lambda_{2}$ further.
} 


\subsection{Extension 1: Multiple Government Spending Shocks (The Complete-Information Case)}

In the benchmark model presented in Section 3, we assume that there is only a single shock to government spending. In this section, we consider both permanent and transitory shocks to government spending. Specifically, we now assume that the government spending-real GDP ratio $g_{t}$ can be expressed as the sum of permanent and transitory components:

$$
g_{t+1}=g_{t+1}^{p}+g_{t+1}^{i},
$$

where the superscripts $p$ and $i$ denote permanent and transitory, respectively. Each of these components follows its own stochastic process; $g_{t}^{p}$ follows a random walk

$$
g_{t+1}^{p}=g_{t}^{p}+\varepsilon_{t+1},
$$

and the transitory component follows a stationary $\mathrm{AR}(1)$ process

$$
g_{t+1}^{i}=\bar{g}+\rho_{g}\left(g_{t}^{i}-\bar{g}\right)+\epsilon_{t+1},
$$

where $\rho_{g} \in[0,1)$, and all innovations are assumed to have zero mean, be uncorrelated over time and with each other, and the variances of $\varepsilon_{t}$ and $\epsilon_{t}$ are $\omega_{\varepsilon}^{2}$ and $\omega_{\epsilon}^{2}$, respectively. We assume that the decomposition of net income, $\left(\omega_{\varepsilon}^{2}, \omega_{\epsilon}^{2}, \rho_{g}\right)$, does not affect the volatility of the change in observed $g\left(\operatorname{sd}\left(\Delta g_{t}\right)\right)$. For simplicity, we consider the case in which $\rho_{g}=0$ and $\omega_{\varepsilon}^{2}=m \omega_{\epsilon}^{2}$ $(m>0)$; allowing $\rho_{g}>0$ does not affect our conclusions but complicates the algebra substantially.

We now derive the expressions for the relative volatility of the change in tax rates to government spending and the correlation between the deficits and the change in government spending. ${ }^{35}$ The following proposition summarizes the results.

Proposition 4 The relative volatility of the change in tax rates to government spending, $\mu$, is:

$$
\mu \equiv \frac{\operatorname{sd}\left(\Delta \tau_{t}\right)}{\operatorname{sd}\left(\Delta g_{t}\right)}=\sqrt{\frac{2(\widetilde{R}-1)^{2}}{(1-\Sigma)[2-\Sigma(1+\widetilde{R})]} /\left[\frac{2+m}{m /(\widetilde{R}-1)^{2}+1 / \widetilde{R}^{2}}\right]},
$$

where we use the facts that $\Delta g_{t+1}=\varepsilon_{t+1}+\epsilon_{t+1}-\epsilon_{t}, \Delta \tau_{t+1}=\left(\rho_{h}-1\right) \tau_{t}+\frac{\widetilde{R}-1}{1-\Sigma} \zeta_{t+1}-(\widetilde{R}-1) \frac{\Sigma \varphi}{1-\Sigma}$, and $\zeta_{t+1}=\frac{\varepsilon_{t+1}}{R-1}+\frac{\epsilon_{t+1}}{R}$. The contemporaneous correlation between the government debt and the change in government spending, corr $\left(\right.$ de $\left.f_{t}, \Delta g_{t}\right)$, is

$$
\operatorname{corr}\left(\operatorname{def}_{t}, \Delta g_{t}\right)=\frac{-m\left(1-\rho_{h}\right) /[(1+n)(\widetilde{R}-1)]+\rho_{h}\left(2-\rho_{h}\right) / R}{\sqrt{2+m} \sqrt{\left(\frac{1}{R}\right)^{2}+\frac{1}{(1+n)^{2}}\left[\frac{2}{(\widetilde{R}-1)^{2}}+\frac{1}{\widetilde{R}^{2}}\right]\left(\frac{1-\rho_{h}}{1+\rho_{h}}\right)-\frac{2\left(1-\rho_{h}\right)}{R^{2}}}},
$$

\footnotetext{
${ }^{35}$ Note that here we use corr $\left(d e f_{t}, \Delta g_{t}\right)$ instead of corr $\left(d e f_{t}, g_{t}\right)$ because in this case the government spending process is non-stationary and thus corr $\left(\operatorname{def}_{t}, g_{t}\right)$ is not well-defined.
} 
where we use the fact that de $f_{t}=\frac{1}{1+n} \frac{1}{\widetilde{R}}\left(g_{t}^{i}-\bar{g}\right)-\left(1-\rho_{h}\right) h_{t}-\frac{1}{1+n} \frac{\Sigma \varphi}{1-\Sigma}$.

Proof. See Online Appendix.

It is clear from (42) that in the FI-RE model where $\Sigma=0, \mu=\sqrt{\left[m+\left(\frac{\widetilde{R}-1}{\widetilde{R}}\right)^{2}\right] /(m+2)}<1$, which is inconsistent with the empirical counterpart reported in Table 1 where $\mu=1.18$. Figure 5 illustrates how this relative volatility varies with the degree of model uncertainty, $\Sigma$, for different values of $m$ that measure the relative importance of the permanent shock $(\varepsilon)$ to the transitory shock $(\epsilon)$. It clearly shows that this ratio is consistently increasing with $\Sigma$, which means that incorporating RB into the multiple-shock case has the potential to help improve the model's predictions. For example, when $m=1$ and $\Sigma=0.52, \mu=1.21$, which is very close to its empirical counterpart.

It is straightforward to show that (42) reduces to $1 / \sqrt{2+m}$ as $\Sigma$ converges to 0 . Figure 6 illustrates how RB affects the correlation between the government debt and government spending for different values of $m$. It is clear that corr $\left(d e f_{t}, \Delta g_{t}\right)$ is consistently decreasing with $\Sigma$ in the multiple-shock case, which means that RB can also have the potential to help explain this dimension. For example, when $m=1$ and $\Sigma=0.1$, corr $\left(d e f_{t}, \Delta g_{t}\right)=0.2$, which is much closer to its empirical counterpart, 0.05, reported in Table 1. (Note that in the FI-RE case $\operatorname{corr}\left(d e f_{t}, \Delta g_{t}\right)=0.6$.

It is worth noting that when the permanent component becomes more and more important than the transitory component, i.e., when $m$ is increasing, $\mu$ converges to 1 in the FI-RE case, and incorporating $\Sigma>0$ can help improve the model's prediction by further increasing $\mu$ in this case. To evaluate the relative importance of the permanent component to the transitory component, we estimate the $g$ process using the U.S. data from the period of $1960-2007$, and find that $\omega_{\epsilon} / \omega_{\varepsilon} \cong 0.1$ or $m \cong 100 .{ }^{36}$ Clearly, the permanent component dominates the transitory component in the $g$ process. Using this estimated value of $m$, it is clear that under FI-RE, $\mu=0.99$ and $\operatorname{corr}\left(d e f_{t}, \Delta g_{t}\right)=0.1$. When $\Sigma=0.16, \mu$ increases to 1.18 and corr $\left(d e f_{t}, \Delta g_{t}\right)$ reduced to -0.05 , which match the corresponding empirical counterparts (1.18 and 0.05$)$ better. $^{37}$

\subsection{Extension 2: Multiple Government Spending Shocks (The Incomplete- Information Case)}

In the above complete-information case, we assume that the government can distinguish the two components in the $g$ process. We now consider another case in which the government cannot dis-

\footnotetext{
${ }^{36}$ The estimation result is obtained using the Matlab toolbox: SSMMATLAB. Using alternative estimation routines do not change the result.

${ }^{37}$ It is important to note that our estimate of $m$ is very imprecise: the one-standard-error confidence interval for $m$ is $[1.2, \infty)$. Therefore, the fact that we use a different value for $\Sigma$ to illustrate our results here will cause no problems for the benchmark model with $\Sigma=0.96$; there is a defensible value of $m$ that would be consistent with almost any value of $\Sigma$ that can match the relevant facts.
} 
tinguish the two components in the spending-to-output ratio specified in (38)-(40). ${ }^{38}$ Specifically, following Pischke (1995), given that the change in $g$ is

$$
\Delta g_{t+1}=\varepsilon_{t+1}+\epsilon_{t+1}-\epsilon_{t},
$$

where $\varepsilon_{t+1}$ and $\epsilon_{t+1}$ are assumed to have zero mean, be uncorrelated over time and with each other, and the variance of $\varepsilon_{t}$ and $\epsilon_{t}$ are $\omega_{\varepsilon}^{2}$ and $\omega_{\epsilon}^{2}$, respectively. The best forecast is to recognize that $g_{t+1}$ is a moving-average process of order one:

$$
\Delta g_{t+1}=\eta_{t+1}-\alpha \eta_{t}
$$

where the innovation, $\eta_{t}$, with mean 0 and variance $\omega_{\eta}^{2}$, is not a fundamental driving process it contains information on current and lagged permanent and transitory shocks to government spending. Equating the variances and autocorrelation coefficients of the original and derived processes (43) and (44), we have

$$
\omega_{\eta}^{2}=\frac{\omega_{\epsilon}^{2}}{\alpha}, \alpha=-\frac{1-\sqrt{1-4 \varrho^{2}}}{2 \varrho}
$$

where $\varrho=-\frac{\omega_{\epsilon}^{2}}{\omega_{\varepsilon}^{2}+2 \omega_{\epsilon}^{2}}$ and $\alpha \in[0,1]$ will be large if the variance of the transitory shock $\omega_{\epsilon}^{2}$ is large relative to the variance of the permanent shock $\omega_{\varepsilon}^{2}$ and will converge to 0 as $\omega_{\epsilon}^{2}$ approaches 0 . As in the last subsection, if we assume that $\omega_{\varepsilon}^{2}=m \omega_{\epsilon}^{2}(m>0), \varrho=-\frac{1}{2+m}>-0.5$.

Following the same state-space reduction procedure used in solving the benchmark model, the new state transition equation can be written as:

$$
h_{t+1}=\widetilde{R} h_{t}-\frac{1}{1+n} \tau_{t}+\widetilde{\zeta}_{t+1}
$$

where $h_{t}=b_{t}+\frac{1}{(1+n)(\widetilde{R}-1)}\left(g_{t}^{p}-\frac{\alpha}{\widetilde{R}} \eta_{t}\right)$ and $\widetilde{\zeta}_{t+1}=\frac{\widetilde{R}-\alpha}{(1+n)(\widetilde{R}-1) \widetilde{R}} \eta_{t+1}$. In this case, an agent with a preference for robustness considers a range of models surrounding the given approximating model, (45):

$$
h_{t+1}=\widetilde{R} h_{t}-\frac{1}{1+n} \tau_{t}+\widetilde{\zeta}_{t+1}+\omega_{\widetilde{\zeta}} \nu_{t},
$$

where $\omega_{\widetilde{\zeta}}=\frac{\widetilde{R}-\alpha}{(1+n)(\widetilde{R}-1)} \omega_{\eta}$. The dynamics of tax and deficit can thus be written as

$$
\begin{aligned}
& \tau_{t+1}=\rho_{h} \tau_{t}+\frac{1-\alpha}{1-\Sigma} \eta_{t+1}-(\widetilde{R}-1) \frac{\Sigma \varphi}{1-\Sigma}, \\
& d e f_{t}=-\left(1-\rho_{h}\right) h_{t}-\frac{1}{1+n} \frac{\Sigma \varphi}{1-\Sigma}+\frac{\alpha}{R} \eta_{t},
\end{aligned}
$$

respectively. The following proposition summarizes the results.

\footnotetext{
${ }^{38}$ We are grateful to an anonymous referee for suggesting we consider this case. As in the first extension, here we also assume that $\rho_{g}=0$.
} 
Proposition 5 The relative volatility of the change in tax rates to government spending, $\mu$, is

$$
\mu \equiv \frac{\operatorname{sd}\left(\Delta \tau_{t}\right)}{\operatorname{sd}\left(\Delta g_{t}\right)}=\sqrt{\frac{2}{(1-\Sigma)[2-\Sigma(1+\widetilde{R})]}\left(\frac{\widetilde{R}-\alpha}{\widetilde{R}}\right)^{2} /\left(1+\alpha^{2}\right)},
$$

and the contemporaneous correlation between the government debt and government spending, $\operatorname{corr}\left(d e f_{t}, g_{t}\right)$, is

$$
\operatorname{corr}\left(\operatorname{def}_{t}, \Delta g_{t}\right)=\frac{\left(\alpha \rho_{h}-1\right)\left(1-\rho_{h}\right) \frac{\widetilde{R}-\alpha}{\widetilde{R}(\widetilde{R}-1)}+\frac{\alpha}{R}}{\sqrt{\frac{\alpha}{R^{2}}+\frac{1-\rho_{h}}{1+\rho_{h}} \frac{\widetilde{R}-\alpha}{\alpha \widetilde{R}(\widetilde{R}-1)}-2 \frac{\left(1-\rho_{h}\right)(\widetilde{R}-\alpha)}{\widetilde{R}^{2}(\widetilde{R}-1)}} \sqrt{\left(1+\alpha^{2}\right) \alpha}} .
$$

Proof. See Online Appendix.

Using (46), it is straightforward to show that when $\Sigma=0, \mu=\frac{\widetilde{R}-\alpha}{\widetilde{R}} \sqrt{\frac{1}{1+\alpha^{2}}}<1$, which is again not consistent with the empirical value of 1.18. We can also see from (46) that this relative volatility is consistently increasing with $\Sigma$, which means that incorporating RB into the incomplete-information case again helps improve the model's predictions. Figure 7 illustrates how this relative volatility varies with the degree of model uncertainty, $\Sigma$, for different values of $m$ that measure the relative importance of the permanent shock $(\varepsilon)$ to the transitory shock $(\epsilon)$.

It is clear from (47) that the correlation converges to $1 / \sqrt{1+\alpha^{2}}$ as $\Sigma$ converges to 0 . Figure 8 illustrates how RB affects the correlation between the government debt and government spending for different values of $m$. It clearly shows that $\operatorname{corr}\left(d e f_{t}, \Delta g_{t}\right)$ is consistently decreasing with $\Sigma$, so as in the benchmark we find incorporating robustness helps resolve the differences between the model and the data.

As $m$ increases to $\infty$, both $\mu$ and corr $\left(d e f_{t}, \Delta g_{t}\right)$ converge to 1 . Permitting $\Sigma>0$ increases $\mu$ and reduces corr $\left(d e f_{t}, \Delta g_{t}\right)$, and thus makes the model fit the data better. Using the estimated $g$ process reported in the last subsection $\left(\omega_{\epsilon} / \omega_{\varepsilon} \cong 0.1\right.$ or $\left.m \cong 100\right)$, we can see that the quantitative effect of incomplete information on the key moments is not significant. ${ }^{39}$ Specifically, when $m=100, \alpha=0.01$, which implies that under FI-RE, $\mu=0.99$ and $\operatorname{corr}\left(\operatorname{de} f_{t}, \Delta g_{t}\right)=1$. When $\Sigma$ increases from 0 to $0.16, \mu$ increases to 1.18 and $\operatorname{corr}\left(d e f_{t}, \Delta g_{t}\right)$ reduced to -0.05 , which match the corresponding empirical counterparts (1.18 and 0.05) better.

\subsection{Extension 3: Shock to Output Growth}

In the benchmark model, we assume that output growth is constant. In this section, we consider an extension in which the growth rate of real GDP follows a stochastic process. The government budget constraint becomes

$$
\left(1+n_{t+1}\right) b_{t+1}=R b_{t}+g_{t}-\tau_{t}
$$

\footnotetext{
${ }^{39}$ Again, we note that our estimate of $m$ is very imprecise but does not contain any values less than 1.
} 
where $n_{t+1}$ follows an $\operatorname{AR}(1)$ process

$$
n_{t+1}-\bar{n}=\rho_{n}\left(n_{t}-\bar{n}\right)+\eta_{t+1},
$$

where $\eta_{t+1}$ is an iid shock with mean zero and variance $\omega_{\eta}^{2}$, and $\bar{n}$ is the steady state growth rate of real GDP. To maintain the model within the LQG setting, we approximate the GBC around the steady state as follows:

$$
(1+\bar{n}) b_{t+1}+\bar{b} n_{t+1}=R b_{t}+g_{t}-\tau_{t}
$$

where $\bar{b}$ is the steady state debt-to-output ratio.

The relative volatility of the change in tax rates to government spending, $\mu$, can be written as

$$
\mu \equiv \frac{\operatorname{sd}\left(\Delta \tau_{t}\right)}{\operatorname{sd}\left(\Delta g_{t}\right)}=\frac{\widetilde{R}-1}{\widetilde{R}-\rho_{g}} \sqrt{\frac{1+\rho_{g}}{2}\left[1+(\bar{b} \widetilde{R})^{2}\left(\frac{\widetilde{R}-\rho_{g}}{\widetilde{R}-\rho_{n}}\right)^{2} \frac{\omega_{\eta}^{2}}{\omega_{\epsilon}^{2}}\right]}
$$

Using the U.S. data from $1960-2007$, we estimate that $\rho_{n}=0.607, \rho_{g}=0.52, \omega_{\eta}=0.017$, $\omega_{\epsilon}=0.006$, and $\bar{b}=0.363$. Given that $\widetilde{R}=1.01$, the FI-RE model predicts that $\mu=0.031$, which means that the model with a shock to output growth is still inconsistent with Table 1 where $\mu=1.18$. Furthermore, the contemporaneous correlation between the government debt and government spending is

$$
\operatorname{corr}\left(d e f_{t}, g_{t}\right)=\frac{1 /\left(1+\rho_{g}\right)}{\sqrt{1 /\left(1-\rho_{g}^{2}\right)} \sqrt{\Omega_{g}+\Omega_{n}}}
$$

where $\Omega_{g}=\frac{1-\rho_{g}}{1+\rho_{g}}$ and $\Omega_{n}=\bar{b}^{2}\left(\frac{\widetilde{R}-\rho_{g}}{\widetilde{R}-\rho_{n}}\right)^{2}\left[\frac{\rho_{n}^{2}\left(1-\rho_{n}\right)}{1+\rho_{n}}+\left(\widetilde{R}-\rho_{n}\right)^{2}\right] \frac{\omega_{\eta}^{2}}{\omega_{\epsilon}^{2}}$. Given these values, we compute that corr $\left(d e f_{t}, g_{t}\right)=0.024$, which is much lower than the empirical counterpart, 0.56 (computed using the HP filter).

The following proposition summarizes the key results when we add robustness to the model with output growth shocks.

Proposition 6 The relative volatility of the change in tax rates to government spending, $\mu$, is

$$
\mu \equiv \frac{\operatorname{sd}\left(\Delta \tau_{t}\right)}{\operatorname{sd}\left(\Delta g_{t}\right)}=\left(\frac{\widetilde{R}-1}{1-\Sigma}\right)\left(\frac{1}{\widetilde{R}-\rho_{g}}\right) \sqrt{\frac{1+\rho_{g}}{1+\rho_{h}}\left[1+(\bar{b} \widetilde{R})^{2}\left(\frac{\widetilde{R}-\rho_{g}}{\widetilde{R}-\rho_{n}}\right)^{2} \frac{\omega_{\eta}^{2}}{\omega_{\epsilon}^{2}}\right]}
$$

and the contemporaneous correlation between the government debt and government spending, $\operatorname{corr}\left(d e f_{t}, g_{t}\right)$, is

$$
\operatorname{corr}\left(\operatorname{def} f_{t}, g_{t}\right)=\frac{1 /\left(1+\rho_{g}\right)-\left(1-\rho_{h}\right) /\left(1-\rho_{g} \rho_{h}\right)}{\sqrt{1 /\left(1-\rho_{g}^{2}\right)} \sqrt{\Omega_{g}+\Omega_{n}}},
$$

where $\Omega_{g}=\frac{1-\rho_{g}}{1+\rho_{g}}+\frac{1-\rho_{h}}{1+\rho_{h}}-2 \frac{\left(1-\rho_{g}\right)\left(1-\rho_{h}\right)}{1-\rho_{g} \rho_{h}}$ and $\Omega_{n}=\bar{b}^{2}\left(\frac{\widetilde{R}-\rho_{g}}{\widetilde{R}-\rho_{n}}\right)^{2}\left[\frac{\rho_{n}^{2}\left(1-\rho_{n}\right)}{1+\rho_{n}}+\left(\widetilde{R}-\rho_{n}\right)^{2}+\frac{1-\rho_{h}}{1+\rho_{h}} \widetilde{R}^{2}-\frac{2 \rho_{n} \widetilde{R}\left(1-\rho_{n}\right)\left(1-\rho_{h}\right)}{1-\rho_{n} \rho_{h}}\right.$ 
Proof. See Online Appendix.

Expression (50) clearly shows that $\mu$ is consistently increasing with $\Sigma$ since $\partial \rho_{h} / \partial \Sigma<0$, which means that incorporating RB into this multiple-shock case has similar effect on the relative volatility as in the benchmark case. Comparing (51) with (28), it is clear that the only difference is the additional term, $\Omega_{n}$, in (51), and RB has the same effect on $\Omega_{n}$ as on $\Omega_{g}$. Therefore, incorporating $\mathrm{RB}$ into this multiple-shock case can also have the potential to help explain this dimension. Using the same parameter values provided above, Figure 9 clearly shows that RB can significantly improve the model's predictions on the relative volatility and the correlation.

\section{Conclusions}

This paper has reconsidered the tax-smoothing hypothesis model of Barro (1979) under the assumption that the government faces model uncertainty regarding the stochastic process for required government spending. Our key finding is that many of the aspects of the tax-smoothing model that are inconsistent with the data under rational expectations - that is, under the assumption of no model uncertainty - are potentially consistent with the data when decisions by the government are made with an eye on robustness. In particular, we can increase the volatility of tax changes relative to changes in spending, decrease the correlation between spending and deficits, and pass VAR tests regarding the joint dynamics of changes in spending and deficits. Finally, we find that the main results obtained in the benchmark model also hold in the extensions with multiple shocks to the government spending to real GDP ratio.

\section{Appendix}

\subsection{Solving the FI-RE TSH Model}

The Lagrangian function for the optimization problem specified in (1) and (3) can be written as

$$
L=E_{0}\left\{\sum_{t=0}^{\infty} \beta^{t}\left[-\frac{1}{2}\left(\tau_{t}+\varphi\right)^{2}-\lambda_{t}\left(R b_{t}+g_{t}-\tau_{t}-(1+n) b_{t+1}\right)\right]\right\}
$$

The FOCs with respect to $\tau_{t}$ and $b_{t+1}$ are:

$$
\begin{array}{r}
-\left(\tau_{t}+\varphi\right)+\lambda_{t}=0 \\
(1+n) \lambda_{t}-\beta R E_{t}\left[\lambda_{t+1}\right]=0
\end{array}
$$

which means that

$$
\tau_{t}=\frac{\beta R}{1+n} E_{t}\left[\tau_{t+1}\right]+\varphi\left(\frac{\beta R}{1+n}-1\right)
$$

When we impose the condition that $\frac{\beta R}{1+n}=1$, (52) leads to the well-known random walk result of tax rates, $\tau_{t}=E_{t}\left[\tau_{t+1}\right]$. 
The intertemporal budget constraint can be written as

$$
\sum_{j=0}^{\infty}\left(\frac{1+n}{R}\right)^{j} g_{t+j}+R b_{t}=\sum_{j=0}^{\infty}\left(\frac{1+n}{R}\right)^{j} \tau_{t+j}
$$

Taking conditional expectations on both sides gives

$$
\begin{aligned}
& E_{t}\left[R b_{t}+\sum_{j=0}^{\infty}\left(\frac{1+n}{R}\right)^{j} g_{t+j}\right]=\sum_{j=0}^{\infty}\left(\frac{1+n}{R}\right)^{j} E_{t}\left[\tau_{t+j}\right]=\left[\sum_{j=0}^{\infty}\left(\frac{1+n}{R}\right)^{j}\left(\frac{1+n}{\beta R}\right)^{j}\right] \tau_{t} \\
& =\frac{1}{1-(1+n)^{2} /\left(\beta R^{2}\right)} \tau_{t}
\end{aligned}
$$

and the optimal tax rate can be written as

$$
\tau_{t}=\left(1-\frac{(1+n)^{2}}{\beta R^{2}}\right) E_{t}\left[R b_{t}+\sum_{j=0}^{\infty}\left(\frac{1+n}{R}\right)^{j} g_{t+j}\right] .
$$

In the case in which $n=0$ and $\beta R=1$,

$$
\tau_{t}=(R-1) E_{t}\left[b_{t}+\frac{1}{R} \sum_{j=0}^{\infty}\left(\frac{1}{R}\right)^{j} g_{t+j}\right]
$$

After defining $h_{t}=E_{t}\left[b_{t}+\frac{1}{R} \sum_{j=0}^{\infty}\left(\frac{1+n}{R}\right)^{j} g_{t+j}\right]$ and combining this expression with the original budget constraint, we obtain the following budget constraint:

$$
(1+n) h_{t+1}=R h_{t}-\tau_{t}+\zeta_{t+1} \text { or } h_{t+1}=\frac{R}{1+n} h_{t}-\frac{1}{1+n} \tau_{t}+\frac{1}{1+n} \zeta_{t+1},
$$

where $\zeta_{t+1}=\sum_{j=0}^{\infty}\left(\frac{1+n}{R}\right)^{j+1}\left(E_{t+1}-E_{t}\right)\left[g_{t+1+j}\right]$.

\subsection{The Equivalence between the Univariate and Multivariate RB Models}

The solution methods used to solve the univariate model and the standard multivariate model are different in the sense that the expectation operator applies to different objects. In the univariate model, the evil agent distorts the transition equation of $h_{t}$ which by itself includes the expectation on future income, whereas in the multivariate model the evil agent distorts the government spending process $\left(g_{t}\right)$. Note that in this case the evil agent does not distort the law of motion for $b_{t}$ because it is a predetermined variable with no uncertainty given $\tau_{t}$. Specifically, the following compact matrix equation can be used to characterize the dynamics of $(b, g)$ :

$$
\left[\begin{array}{c}
b_{t+1} \\
g_{t+1}
\end{array}\right]=\left[\begin{array}{ll}
\widetilde{R} & \frac{1}{1+n} \\
0 & \rho_{g}
\end{array}\right]\left[\begin{array}{c}
b_{t} \\
g_{t}
\end{array}\right]-\left[\begin{array}{l}
\frac{1}{1+n} \\
0
\end{array}\right] \tau_{t}+\left[\begin{array}{l}
0 \\
1
\end{array}\right] \epsilon_{t+1},
$$


where we ignore the constant term as it does not affect the stochastic properties of the model. Assume that (53) is the approximation model. The corresponding distortion model is

$$
\left[\begin{array}{l}
b_{t+1} \\
g_{t+1}
\end{array}\right]=\left[\begin{array}{ll}
\widetilde{R} & \frac{1}{1+n} \\
0 & \rho_{g}
\end{array}\right]\left[\begin{array}{l}
b_{t} \\
g_{t}
\end{array}\right]-\left[\begin{array}{l}
\frac{1}{1+n} \\
0
\end{array}\right] \tau_{t}+\left[\begin{array}{l}
0 \\
1
\end{array}\right]\left(\epsilon_{t+1}+\omega \nu_{t}\right) .
$$

The robust control problem can thus be written as follows:

$$
v\left(b_{t}, g_{t}\right)=\min _{\tau_{t}} \max _{\nu_{t}}\left\{-\frac{1}{2} \tau_{t}^{2}+\beta\left[\vartheta \nu_{t}^{2}+E_{t}\left[v\left(b_{t+1}, g_{t+1}\right)\right]\right]\right\},
$$

subject to (54). Since there is no closed-form solution for this problem, we solve this model numerically. Following the standard procedure proposed in Hansen and Sargent (Chapters 2 and 10, 2007), we can solve this robust linear-quadratic regulator numerically and obtain a linear function of $\tau_{t}$ in terms of $\left(b_{t}, g_{t}\right)$ :

$$
\tau_{t}=\operatorname{MPT}\left(b_{t}+\lambda g_{t}\right)
$$

where MPT is the marginal propensity to tax, $\lambda$ measures the relative importance of government spending $(g)$ and government debts $(b)$ in determining optimal taxation. From 55 ), it is clear that in the multivariate model RB might affect the relative importance of the two state variables on the taxation function, while in the univariate model the relative importance of the two effects are fixed in order to reduce the state space. (i.e., $h_{t}=b_{t}+\frac{1}{(1+n)\left(\widetilde{R}-\rho_{g}\right)} g_{t}+\frac{\left(1-\rho_{g}\right) \bar{g}}{(1+n)\left(\widetilde{R}-\rho_{g}\right)(\widetilde{R}-1)}$.) We now show that numerically the two modeling strategies can lead to the identical decision rules. Note that in our univariate model, after introducing the new state variable, the consumption function under RB can be written as

$$
\tau_{t}=\operatorname{MPT} h_{t}
$$

where we ignore the constant term; MPT $=\frac{(1+n)(\widetilde{R}-1)}{1-\Sigma}, h_{t}=b_{t}+\frac{1}{(1+n)\left(\widetilde{R}-\rho_{g}\right)} g_{t}$, and $\Sigma=$ $\widetilde{R} \omega_{\zeta}^{2} /(2 \vartheta)$. Figure 10 illustrates how the marginal propensity of taxation (MPT) is affected by the degree of robustness in both the multivariate and univariate models when we set $\widetilde{R}=1.01$, $n=3 \%, \rho_{g}=0.6$, and $\omega_{\epsilon}^{2}=1$. The figure clearly shows that the two models deliver the identical MPT for various values of $\vartheta$. For example, the multivariate model predicts that

$$
\tau_{t}=0.0182 b_{t}+0.0444 g_{t}
$$

when $1 / \vartheta=0.15$, whereas the univariate model predicts that

$$
\tau_{t}=0.0182 h_{t}=0.0182\left(b_{t}+2.4390 g_{t}\right)=0.0182 b_{t}+0.0444 g_{t}
$$

for the same value of $\vartheta$ (i.e., $\Sigma=0.4337$ ). In addition, we find the relative importance of government spending and government debt is also identical in the two models. (See Figure 11 for an illustration.) 


\subsection{Deriving the Standard Errors for the VAR Test}

In this appendix we derive expressions for the standard errors of the estimators for $\lambda_{1}$ and $\lambda_{2}$ used in the VAR test of Section 4.4. Let $d \equiv 1 / \tilde{R}$,

$$
A \equiv\left[\begin{array}{ll}
a_{11} & a_{12} \\
a_{21} & a_{22}
\end{array}\right],
$$

and $I$ be the $2 \times 2$ identify matrix. Define

$$
\Lambda \equiv\left[\begin{array}{ll}
\lambda_{1} & \lambda_{2}
\end{array}\right]=-\left[\begin{array}{ll}
1 & 0
\end{array}\right] A d(I-d A)^{-1} .
$$

Define

$$
B \equiv(I-d A)^{-1}=\left[\begin{array}{ll}
b_{11} & b_{12} \\
b_{21} & b_{22}
\end{array}\right],
$$

we have $B(I-d A)=I$, and we can solve for the coefficients of the $B$ matrix:

$$
\begin{aligned}
& b_{11}=\frac{1-d a_{22}}{\left(1-d a_{11}\right)\left(1-d a_{22}\right)-d^{2} a_{12} a_{21}}, \\
& b_{12}=\frac{d a_{12}}{\left(1-d a_{11}\right)\left(1-d a_{22}\right)-d^{2} a_{12} a_{21}}, \\
& b_{21}=\frac{d a_{21}}{\left(1-d a_{11}\right)\left(1-d a_{22}\right)-d^{2} a_{12} a_{21}}, \\
& b_{22}=\frac{1-d a_{11}}{\left(1-d a_{11}\right)\left(1-d a_{22}\right)-d^{2} a_{12} a_{21}} .
\end{aligned}
$$

Now we substitute the $B$ matrix into Equation (57), obtaining

$$
\begin{aligned}
& \lambda_{1}=-d \frac{a_{11}\left(1-d a_{22}\right)+d a_{12} a_{21}}{\left(1-d a_{11}\right)\left(1-d a_{22}\right)-d^{2} a_{12} a_{21}} \equiv-d \frac{c_{1}}{c_{2}}, \\
& \lambda_{2}=-\frac{d a_{12}}{\left(1-d a_{11}\right)\left(1-d a_{22}\right)-d^{2} a_{12} a_{21}} \equiv-\frac{f_{1}}{f_{2}} .
\end{aligned}
$$

Let the $2 \times 4$ matrix $G$ denote the gradient of $\Lambda$ with respect to the vectorization of VAR coefficient matrix $A: \operatorname{vec}(A) \equiv\left[\begin{array}{llll}a_{11} & a_{21} & a_{12} & a_{22}\end{array}\right]^{T}$, where $G(i, j)=\frac{\partial \lambda_{i}}{\partial \operatorname{vec}(A)_{j}}(i=1,2 ; j=1,2,3,4)$ and $G$ is given by

$$
\begin{aligned}
& G_{11}=-\frac{d}{c_{2}}\left(1-d a_{22}\right)\left(1-\lambda_{1}\right), G_{12}=-\frac{d^{2} a_{12}}{c_{2}}\left(1+d \frac{c_{1}}{c_{2}}\right) \\
& G_{13}=-\frac{d^{2} a_{21}}{c_{2}}\left(1+d \frac{c_{1}}{c_{2}}\right), G_{14}=\frac{d^{2}}{c_{2}}\left(a_{11}-\frac{c_{1}}{c_{2}}\left(1-d a_{11}\right)\right) \\
& G_{21}=\frac{d}{f_{2}} \lambda_{2}\left(1-d a_{22}\right), G_{22}=\frac{d^{2}}{f_{2}} \lambda_{2} a_{12} \\
& G_{23}=-\frac{d}{f_{2}}\left(1-\lambda_{2} d a_{21}\right), G_{24}=\frac{d}{f_{2}} \lambda_{2}\left(1-d a_{11}\right)
\end{aligned}
$$


Finally, the covariance matrix of $\Lambda$ is computed as

$$
\operatorname{var}(\Lambda)=G \operatorname{var}(A) G^{T}
$$

where $\operatorname{var}(A)$ is the covariance matrix $(4 \times 4)$ of $\operatorname{vec}(A)$.

\subsection{Solving the Robust TSH Model}

To solve the Bellman equation (17), we conjecture that

$$
v\left(h_{t}\right)=-A h_{t}^{2}-B h_{t}-C,
$$

where $A, B$, and $C$ are undetermined coefficients. Substituting this guessed value function into the Bellman equation gives

$$
-A h_{t}^{2}-B h_{t}-C=\max _{\tau_{t}} \min _{\nu_{t}}\left\{-\frac{1}{2}\left(\tau_{t}+\varphi\right)^{2}+\beta E_{t}\left[\vartheta \nu_{t}^{2}-A h_{t+1}^{2}-B h_{t+1}-C\right]\right\},
$$

where $h_{t+1}=\widetilde{R} h_{t}-\frac{1}{1+n} \tau_{t}+\widetilde{\zeta}_{t+1}+\omega_{\widetilde{\zeta}} \nu_{t}$. We can do the min and max operations in any order, so we choose to do the minimization first. The first-order condition for $\nu_{t}$ is

$$
2 \vartheta \nu_{t}-2 A E_{t}\left(\widetilde{R} h_{t}-\frac{1}{1+n} \tau_{t}+\omega_{\widetilde{\zeta}} \nu_{t}\right) \omega_{\widetilde{\zeta}}-B \omega_{\widetilde{\zeta}}=0
$$

which means that

$$
\nu_{t}=\frac{B+2 A\left(\widetilde{R} h_{t}-\frac{1}{1+n} \tau_{t}\right)}{2\left(\vartheta-A \omega_{\widetilde{\zeta}}^{2}\right)} \omega_{\widetilde{\zeta}}
$$

Substituting (59) back into (58) gives

$$
-A h_{t}^{2}-B h_{t}-C=\max _{\tau_{t}}\left\{-\frac{1}{2}\left(\tau_{t}+\varphi\right)^{2}+\beta E_{t}\left(\vartheta\left[\frac{B+2 A\left(\widetilde{R} h_{t}-\frac{1}{1+n} \tau_{t}\right)}{2\left(\vartheta-A \omega_{\widetilde{\zeta}}^{2}\right)} \omega_{\widetilde{\zeta}}\right]^{2}-A h_{t+1}^{2}-B h_{t+1}-C\right)\right\}
$$

The first-order condition for $\tau_{t}$ is

$$
\begin{array}{r}
-\left(\tau_{t}+\varphi\right)-2 \beta \vartheta \frac{1}{1+n} \frac{A \omega_{\widetilde{\zeta}}}{\vartheta-A \omega_{\widetilde{\zeta}}^{2}} \nu_{t}+2 \beta A \frac{1}{1+n}\left(1+\frac{A \omega_{\widetilde{\zeta}}^{2}}{\vartheta-A \omega_{\widetilde{\zeta}}^{2}}\right)\left(\widetilde{R} h_{t}-\frac{1}{1+n} \tau_{t}+\omega_{\widetilde{\zeta}} \nu_{t}\right) \\
+\beta B \frac{1}{1+n}\left(1+\frac{A \omega_{\widetilde{\zeta}}^{2}}{\vartheta-A \omega_{\widetilde{\zeta}}^{2}}\right)=0 .
\end{array}
$$

Using the solution for $\nu_{t}$ the solution for taxation is

$$
\tau_{t}=\frac{2 \beta \widetilde{R} A}{(1+n)-(1+n) A \omega_{\widetilde{\zeta}}^{2} / \vartheta+2 \beta A /(1+n)} h_{t}+\frac{-(1+n)\left(1-A \omega_{\widetilde{\zeta}}^{2} / \vartheta\right) \varphi+\beta B}{(1+n)-(1+n) A \omega_{\widetilde{\zeta}}^{2} / \vartheta+2 \beta A /(1+n)} .
$$


Substituting the above expressions into the Bellman equation gives

$$
\begin{aligned}
& -A h_{t}^{2}-B h_{t}-C \\
& =-\frac{1}{2}\left[\frac{2 \beta \widetilde{R} A}{(1+n)-(1+n) A \omega_{\widetilde{\zeta}}^{2} / \vartheta+2 \beta A /(1+n)} h_{t}+\frac{\beta B+2 \beta \varphi A /(1+n)}{(1+n)-(1+n) A \omega_{\widetilde{\zeta}}^{2} / \vartheta+2 \beta A /(1+n)}\right]^{2} \\
& +\frac{\beta \vartheta \omega_{\widetilde{\zeta}}^{2}}{\left[2\left(\vartheta-A \omega_{\widetilde{\zeta}}^{2}\right)\right]^{2}}\left\{\begin{array}{c}
2 A \widetilde{R}\left[\frac{(1+n)^{2}-(1+n)^{2} A \omega_{\widetilde{\zeta}}^{2} / \vartheta}{(1+n)^{2}-(1+n)^{2} A \omega_{\widetilde{\zeta}}^{2} / \vartheta+2 \beta A}\right] h_{t} \\
+\frac{\left((1+n)^{2} B+2 A(1+n) \varphi\right)\left(1-A \omega_{\widetilde{\zeta}}^{2} / \vartheta\right)}{(1+n)^{2}-(1+n)^{2} A \omega_{\widetilde{\zeta}}^{2} / \vartheta+2 \beta A}
\end{array}\right\}^{2} \\
& -\beta A\left\{\begin{array}{c}
{\left[\frac{(1+n)^{2} \widetilde{R}}{(1+n)^{2}-(1+n)^{2} A \omega_{\widetilde{\zeta}}^{2} / \vartheta+2 \beta A}\right]^{2} h_{t}^{2}-\frac{(1+n)^{2} \widetilde{R}\left[-2(1+n) \varphi+2 \beta B-B \omega_{\widetilde{\zeta}}^{2} / \vartheta(1+n)^{2}\right]}{\left[(1+n)^{2}-(1+n)^{2} A \omega_{\widetilde{\zeta}}^{2} / \vartheta+2 \beta A\right]^{2}} h_{t}} \\
+\frac{-2(1+n) \varphi+2 \beta B-B \omega_{\widetilde{\widetilde{\zeta}}}^{2} / \vartheta(1+n)^{2}}{2\left[(1+n)^{2}-(1+n)^{2} A \omega_{\widetilde{\zeta}}^{2} / \vartheta+2 \beta A\right]}+\omega_{\widetilde{\zeta}}^{2}
\end{array}\right\} \\
& -\beta B\left[\frac{(1+n)^{2} \widetilde{R}}{(1+n)^{2}-(1+n)^{2} A \omega_{\widetilde{\zeta}}^{2} / \vartheta+2 \beta A} h_{t}-\frac{-2(1+n) \varphi+2 \beta B-B \omega_{\widetilde{\zeta}}^{2} / \vartheta(1+n)^{2}}{2\left((1+n)^{2}-(1+n)^{2} A \omega_{\widetilde{\zeta}}^{2} / \vartheta+2 \beta A\right)}\right]-\beta C .
\end{aligned}
$$

Given $\beta \widetilde{R}=1$, collecting and matching terms, the constant coefficients turn out to be

$$
\begin{aligned}
A & =\frac{(1+n)^{2} \widetilde{R}(\widetilde{R}-1)}{2-(1+n)^{2} \widetilde{R} \omega_{\widetilde{\zeta}}^{2} / \vartheta}, \\
B & =\frac{(1+n) \varphi \widetilde{R}}{1-(1+n)^{2} \widetilde{R} \omega_{\widetilde{\zeta}}^{2} /(2 \vartheta)}
\end{aligned}
$$

Substituting (61) and (62) into (60) yields the taxation function (18) in the text.

We impose parameter restrictions so that $A>0$, implying the value function is concave; these restrictions amount to requiring that $\vartheta$ not be too small and are shown in the text to imply $\Sigma<1$.

\subsection{Deriving the Stochastic Properties of Optimal Taxation and Government Debts under RB}

\subsubsection{Deriving the Volatility of the Change in Taxes}

Given $(25), \tau_{t+1}=\rho_{h} \tau_{t}+\frac{\widetilde{R}-1}{1-\Sigma} \zeta_{t+1}-(\widetilde{R}-1) \frac{\Sigma \varphi}{1-\Sigma}$, the variance of $\Delta \tau_{t+1}$ can be written as: 


$$
\begin{aligned}
\operatorname{var}\left(\Delta \tau_{t+1}\right) & =\operatorname{var}\left(\left(\rho_{h}-1\right) \tau_{t}+\frac{\widetilde{R}-1}{1-\Sigma} \zeta_{t+1}\right) \\
& =\left(1-\rho_{h}\right)^{2}\left(\frac{\widetilde{R}-1}{1-\Sigma}\right)^{2} \frac{\omega_{\zeta}^{2}}{1-\rho_{h}^{2}}+\left(\frac{\widetilde{R}-1}{1-\Sigma}\right)^{2} \omega_{\zeta}^{2} \\
& =\frac{2}{1+\rho_{h}}\left(\frac{\widetilde{R}-1}{1-\Sigma}\right)^{2} \omega_{\zeta}^{2} .
\end{aligned}
$$

Using the definition of the relative volatility of the change in taxes and government spending, we can obtain (27) in the text.

\subsubsection{Deriving the Correlation between Government Debts and Spending}

Given $(20), d e f_{t}=\frac{1-\rho_{g}}{(1+n)\left(\widetilde{R}-\rho_{g}\right)}\left(g_{t}-\bar{g}\right)-\left(1-\rho_{h}\right) h_{t}-\frac{1}{1+n} \frac{\Sigma \varphi}{1-\Sigma}$,

$$
\begin{aligned}
\operatorname{var}\left(\text { def }_{t}\right) & =\operatorname{var}\left(\frac{1}{1+n} \frac{1-\rho_{g}}{\widetilde{R}-\rho_{g}} g_{t}-\left(1-\rho_{h}\right) h_{t}\right) \\
& =\left(\frac{1-\rho_{g}}{(1+n)\left(\widetilde{R}-\rho_{g}\right)}\right)^{2} \operatorname{var}\left(g_{t}\right)+\left(1-\rho_{h}\right)^{2} \operatorname{var}\left(h_{t}\right)-2 \frac{\left(1-\rho_{g}\right)\left(1-\rho_{h}\right)}{(1+n)\left(\widetilde{R}-\rho_{g}\right)} \operatorname{cov}\left(g_{t}, h_{t}\right) \\
& =\left(\frac{1-\rho_{g}}{\widetilde{R}-\rho_{g}}\right)^{2} \frac{\left(\widetilde{R}-\rho_{g}\right)^{2} \omega_{\widetilde{\zeta}}^{2}}{1-\rho_{g}^{2}}+\left(1-\rho_{h}\right)^{2} \frac{\omega_{\widetilde{\zeta}}^{2}}{1-\rho_{h}^{2}}-2 \frac{\left(1-\rho_{g}\right)\left(1-\rho_{h}\right)}{\widetilde{R}-\rho_{g}} \frac{\left(\widetilde{R}-\rho_{g}\right) \omega_{\widetilde{\zeta}}^{2}}{1-\rho_{g} \rho_{h}} \\
& =\left[\frac{1-\rho_{g}}{1+\rho_{g}}+\frac{1-\rho_{h}}{1+\rho_{h}}-2 \frac{\left(1-\rho_{h}\right)\left(1-\rho_{g}\right)}{1-\rho_{g} \rho_{h}}\right] \omega_{\widetilde{\zeta}}^{2},
\end{aligned}
$$

where we use the facts that $\rho_{h}=1-\frac{\Sigma(\widetilde{R}-1)}{1-\Sigma}, \operatorname{var}\left(g_{t}\right)=\frac{\omega^{2}}{1-\rho_{g}^{2}}=\frac{\left(\widetilde{R}-\rho_{g}\right)^{2} \omega_{\zeta}^{2}}{1-\rho_{g}^{2}}, \operatorname{var}\left(h_{t}\right)=\frac{\omega_{\widetilde{\zeta}}^{2}}{1-\rho_{h}^{2}}$, and $\operatorname{cov}\left(g_{t}, h_{t}\right)=\frac{(1+n)\left(\widetilde{R}-\rho_{g}\right) \omega_{\widetilde{\zeta}}^{2}}{1-\rho_{g} \rho_{h}}$. Using $(20)$ and (10), the covariance between the government debt 
and spending is

$$
\begin{aligned}
\operatorname{cov}\left(\operatorname{def} f_{t}, g_{t}\right) & =\operatorname{cov}\left(\frac{1-\rho_{g}}{(1+n)\left(\widetilde{R}-\rho_{g}\right)} g_{t}-\left(1-\rho_{h}\right) h_{t}, g_{t}\right) \\
& =\frac{1-\rho_{g}}{(1+n)\left(\widetilde{R}-\rho_{g}\right)} \operatorname{var}\left(g_{t}\right)-\left(1-\rho_{h}\right) \operatorname{cov}\left(h_{t}, g_{t}\right) \\
& =\frac{1-\rho_{g}}{(1+n)\left(\widetilde{R}-\rho_{g}\right)} \frac{\left(\widetilde{R}-\rho_{g}\right)^{2}}{1-\rho_{g}^{2}} \omega_{\zeta}^{2}-\frac{(1+n)\left(\widetilde{R}-\rho_{g}\right)\left(1-\rho_{h}\right)}{1-\rho_{g} \rho_{h}} \omega_{\widetilde{\zeta}}^{2} \\
& =(1+n)\left(\widetilde{R}-\rho_{g}\right)\left(\frac{1}{1+\rho_{g}}-\frac{1-\rho_{h}}{1-\rho_{g} \rho_{h}}\right) \omega_{\widetilde{\zeta}}^{2}
\end{aligned}
$$

Using (63) and (64), the correlation between the current account and net income can be written as (28) in the text.

\section{References}

[1] Adam, K., 2004. On the relation between Bayesian and robust decision making. Journal of Economic Dynamics and Control 28(10), 2105-2117.

[2] Adler, J., 2006. The tax-smoothing hypothesis: evidence from Sweden, 1952-1999. Scandinavian Journal of Economics 108(1), 81-95.

[3] Angeletos, G.-M., 2002. Fiscal policy with non-contingent debt and the optimal maturity structure. Quarterly Journal of Economics 117(3), 1105-1131.

[4] Aiyagari, S.R., Marcet, A., Sargent, T.J., Seppala, J., 2002. Optimal taxation without statecontingent debt. Journal of Political Economy 110(6), 1220-1254.

[5] Backus, D.K., Routledge, B.R., Zin, S.E., 2004. Exotic preferences for macroeconomists. NBER Macroeconomics Annual 2004, 319-414.

[6] Barro, R.J., 1979. On the determination of the public debt. Journal of Political Economy 87(5), 940-971.

[7] Barro, R.J., 1981. On the predictability of tax-rate changes. Working Paper 636, National Bureau of Economic Research.

[8] Bidder, R., Smith, M.E., 2012. Robust animal spirits. Journal of Monetary Economics 59(8), 738-750. 
[9] Bohn, H., 1990. Tax smoothing with financial instruments. American Economic Review 80(5), $1217-1230$.

[10] Bohn, H., 1998. The behavior of US public debt and deficits. Quarterly Journal of Economics 113(3), 949-963.

[11] Campbell, J.Y., 1987. Does saving anticipate declining labor income? An alternative test of the permanent income hypothesis. Econometrica 55(6), 1249-1273.

[12] Cashin, P, Haque, N.u.H., Olekalns, N., 2003. Tax smoothing, tax tilting, and fiscal sustainability in Pakistan. Economic Modelling 20(1), 47-67.

[13] Cashin, P., Olekalns, N., Sahay, R., 1998. Tax smoothing in a financially repressed economy: evidence from India. Working Paper 98/172, International Monetary Fund.

[14] Cogley, T., Colacito, R., Hansen, L.P., Sargent, T.J., 2007. Benefits from U.S. monetary policy experimentation in the days of Samuelson and Solow and Lucas. Journal of Money, Credit and Banking, 39 (Suppl.), 67-99.

[15] Dennis, R., 2010. How robustness can lower the cost of discretion. Journal of Monetary Economics 57(6), 653-667.

[16] Ellison, M., Sargent, T.J., 2012. A defence of the FOMC. International Economic Review 53(4), 1047-1065.

[17] Ghosh, A.R., 1995). Intertemporal tax-smoothing and the government budget surplus: Canada and the United States. Journal of Money, Credit and Banking 27(4), 1033-1045.

[18] Giannoni, M., 2002. Does model uncertainty justify caution? Robust optimal monetary policy in a forward-looking model. Macroeconomic Dynamics 6(1), 111-144.

[19] Giordani, P., Soderlind, P., 2004. Solution of macromodels with Hansen-Sargent robust policies: some extensions. Journal of Economic Dynamics and Control 28(12), 2367-2397.

[20] Hansen, L.P., Sargent, T.J., 2007, Robustness, Princeton University Press.

[21] Hansen, L.P., Sargent, T.J., Tallarini, T.D., Jr., 1999. Robust permanent income and pricing. Review of Economic Studies 66(4), 873-907.

[22] Hansen, L.P., Sargent, T.J., Wang, N., 2002. Robust permanent income and pricing with filtering. Macroeconomic Dynamics 6(1), 40-84.

[23] Huang, C.-H., Lin, K.S., 1993. Deficits, government expenditures and tax smoothing in the United States: 1929-1988. Journal of Monetary Economics 31(3), 317-339. 
[24] Jacobson, D.H., 1973. Optimal stochastic linear systems with exponential performance criteria and their relation to deterministic differential games. IEEE Transactions on Automatic Control 18, 124-131.

[25] Karantounias, A.G., 2013. Managing expectations and fiscal policy. Theoretical Economics $8(1), 193-231$.

[26] Kasa, K., 2002. Model uncertainty, robust policies, and the value of commitment. Macroeconomic Dynamics 6(1), 145-166.

[27] Lloyd-Ellis, H., Zhan, S., and Zhu, X. 2002. Tax smoothing with stochastic interest rates: a re-assessment of Clinton's fiscal legacy. Journal of Money, Credit, and Banking 37(4), 699724.

[28] Lucas, R.E., Jr., Stokey, N.L., 1983. Optimal fiscal and monetary policy in an economy without capital. Journal of Monetary Economics 12(1), 55-93.

[29] Luo, Y., 2008. Consumption dynamics under information processing constraints. Review of Economic Dynamics 11(2), 366-385.

[30] Luo, Y., Young, E.R., 2010. Risk-sensitive consumption and savings under rational inattention. American Economic Journal: Macroeconomics 2(4), 281-325.

[31] Luo, Y., Young, E.R., 2014. Induced Uncertainty, Market Price of Risk, and the Dynamics of Consumption and Wealth. Working Paper, University of Hong Kong and University of Virginia.

[32] Luo, Y., Nie, J., Young, E.R., 2012. Robustness, information-processing constraints, and the current account in small open economies. Journal of International Economics 88(1), 104-120.

[33] Luo, Y., Nie, J., Young, E.R., 2014. Robust control, informational frictions, and international consumption correlations. European Economic Review 67(1), 1-27.

[34] Olekalns, N., 1997. Australian evidence on tax smoothing and the optimal budget surplus. Economic Record 73(222), 248-257.

[35] Onatski, A.,Williams, N., 2003. Modeling model uncertainty. Journal of the European Economic Association 1(5), 1087-1122.

[36] Pischke, J.S., 1995. Individual income, incomplete information, and aggregate consumption. Econometrica 63(4), 805-840.

[37] Roubini, N., 1988. Current account and budget deficits in an intertemporal model of consumption and taxation smoothing. A solution to the Feldstein-Horioka puzzle? Working Paper No. 2773, National Bureau of Economic Research. 
[38] Sahasakul, C., 1986. The U.S. evidence on optimal taxation over time. Journal of Monetary Economics 18(3), 251-275.

[39] Sargent, T.J., 2001. Comment on 'fiscal consequences for Mexico adopting the dollar' by Christopher A. Sims. Journal of Money, Credit, and Banking 33(2), 617-625.

[40] Strazicich, M.C., 1997. Does tax smoothing differ by the level of government? Time series evidence from Canada and the United States. Journal of Macroeconomics 19(2), 305-326.

[41] Svec, J., 2012. Optimal fiscal policy with robust control. Journal of Economic Dynamics and Control 36(3), 349-368.

[42] Svensson, L.E.O., Williams, N., 2007. Monetary policy with model uncertainty: distribution forecast targeting. Working Paper 11733, National Bureau of Economic Research.

[43] Trehan, B., Walsh, C.E., 1990. Seigniorage and tax smoothing in the United States 1914-1986. Journal of Monetary Economics 25(1), 97-112.

[44] van der Ploeg, F., 1993. A closed-form solution for a model of precautionary saving. Review of Economic Studies 60(2), 385-395.

[45] Whittle, P., 1981. Risk-sensitive linear/quadratic/Gaussian control. Advances in Applied Probability 13, 764-777.

[46] Woodford, M., 2010. Robustly optimal monetary policy with near-rational expectations. American Economic Review 100(1), 274-303.

[47] Young, E.R., 2012. Robust policymaking in the face of sudden stops. Journal of Monetary Economics 59(5), 512-527. 

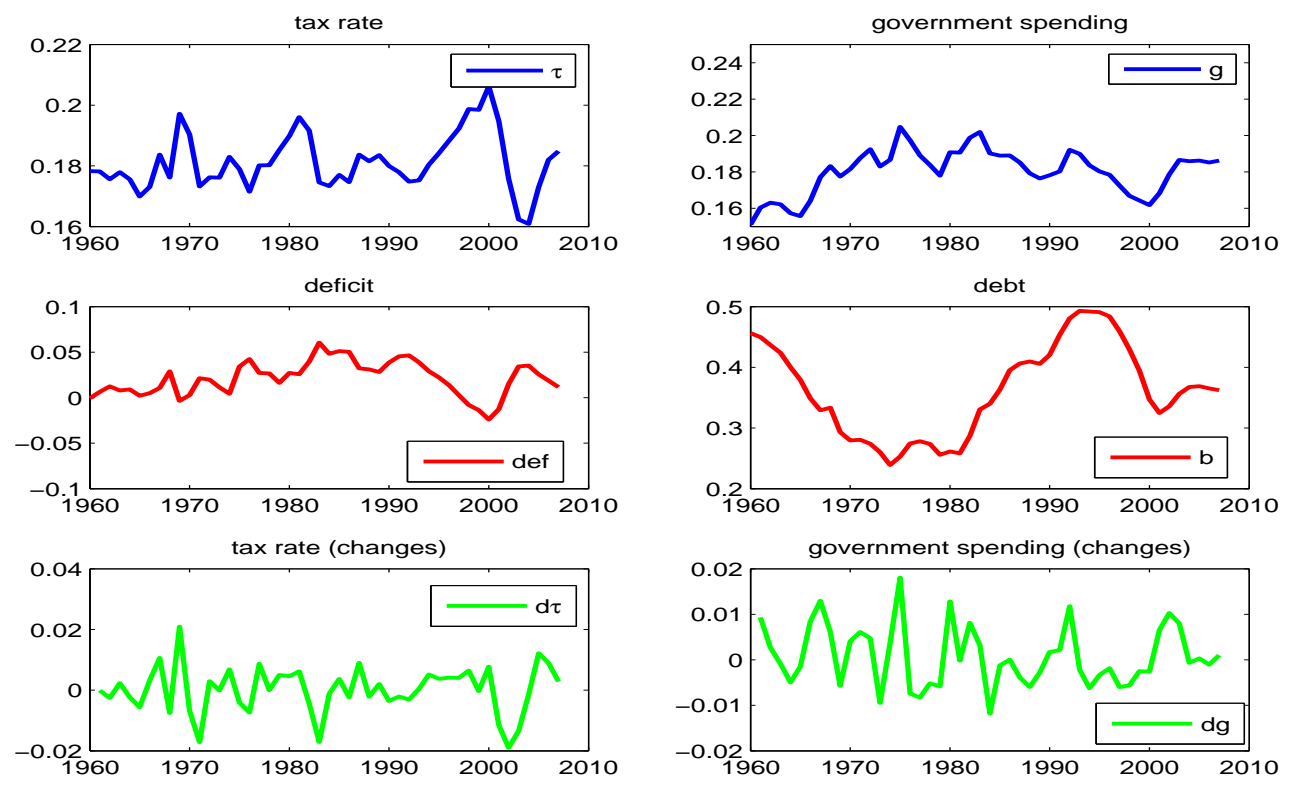

Figure 1: US Fiscal Policy 


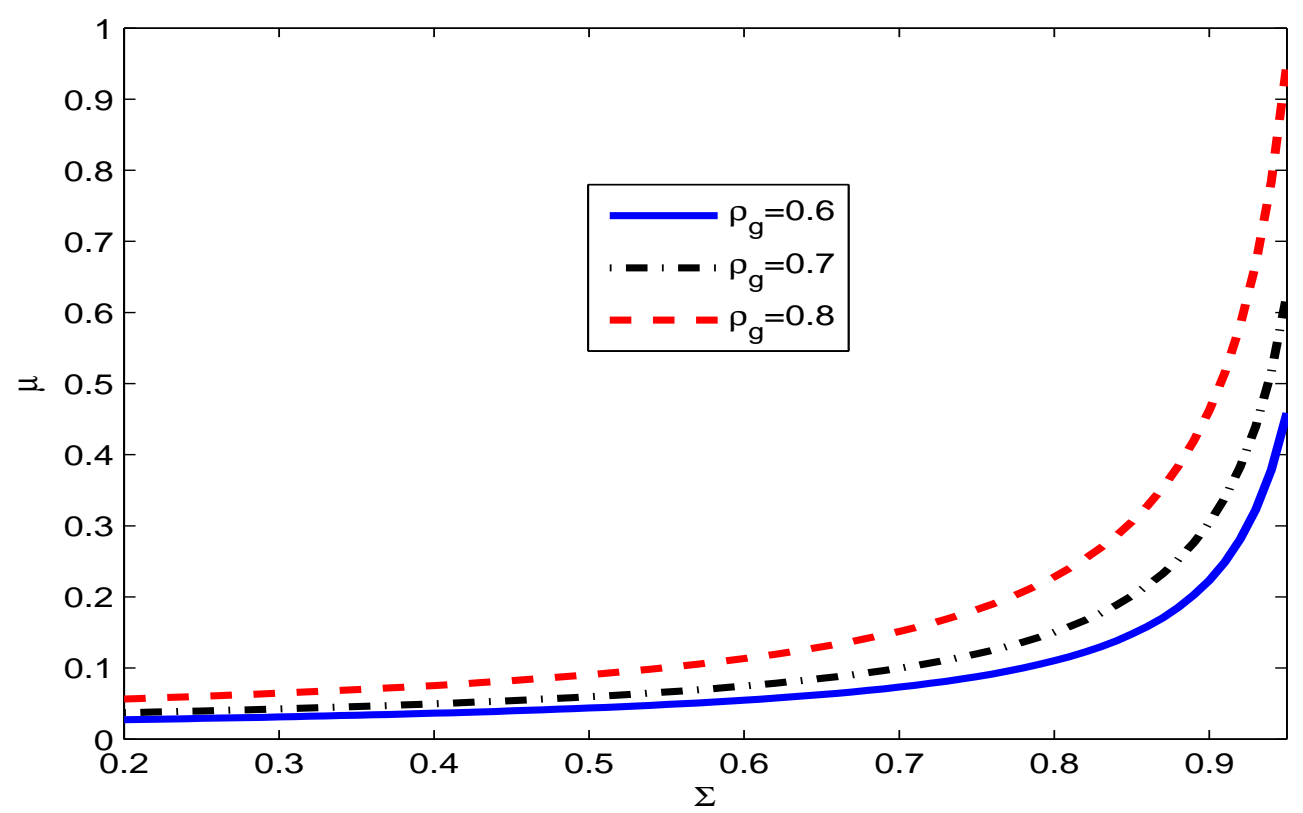

Figure 2: The Relative Volatility of Tax Change to Government Spending

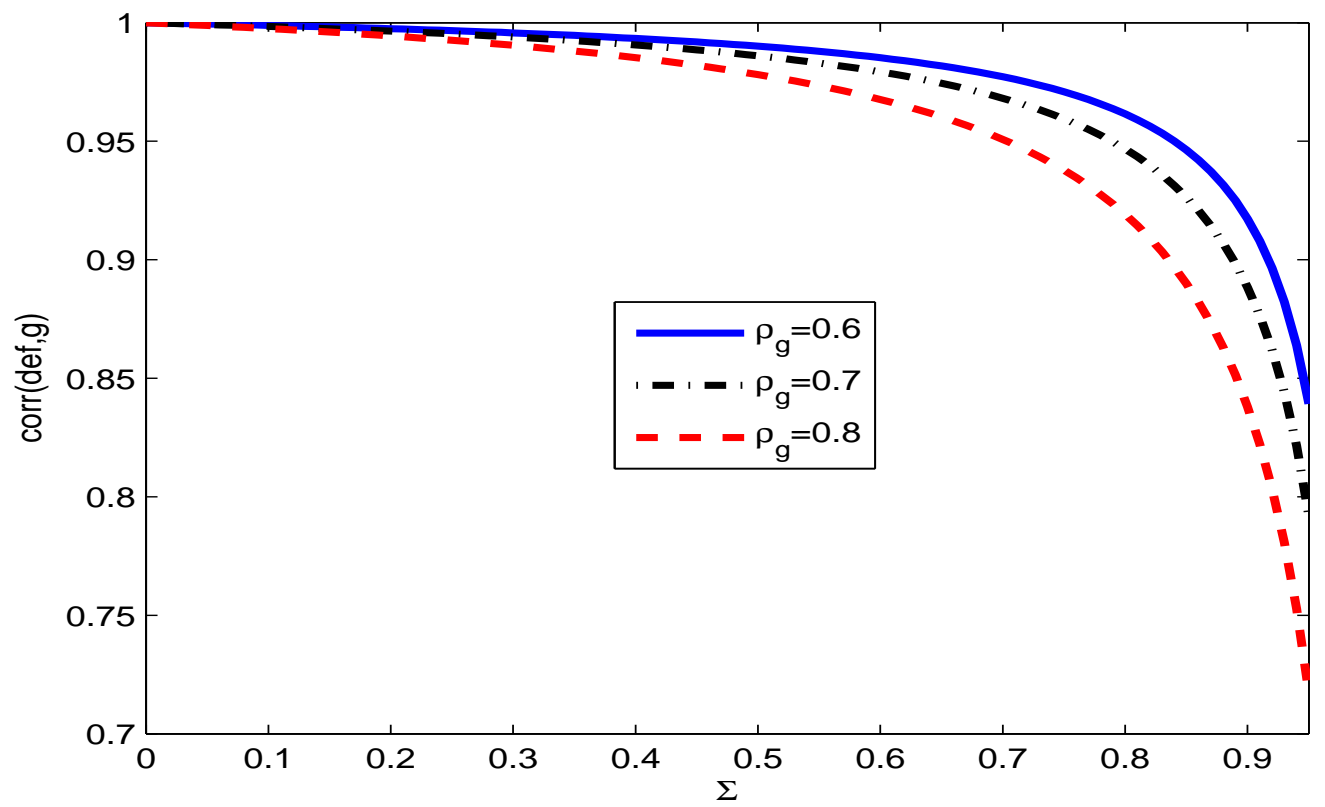

Figure 3: The Correlation between the Government Deficit and Government Spending 


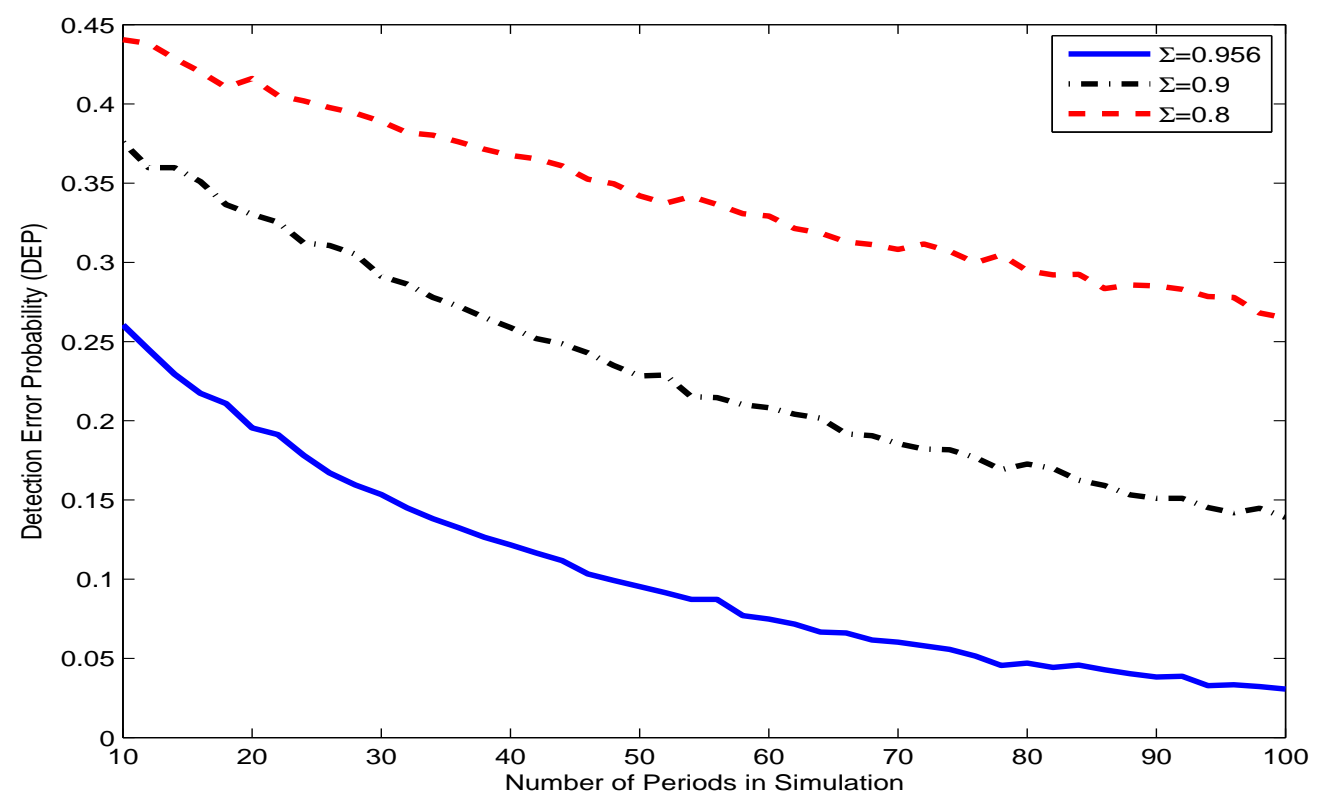

Figure 4: Detection Error Probability (DEP) and the Simulation Length

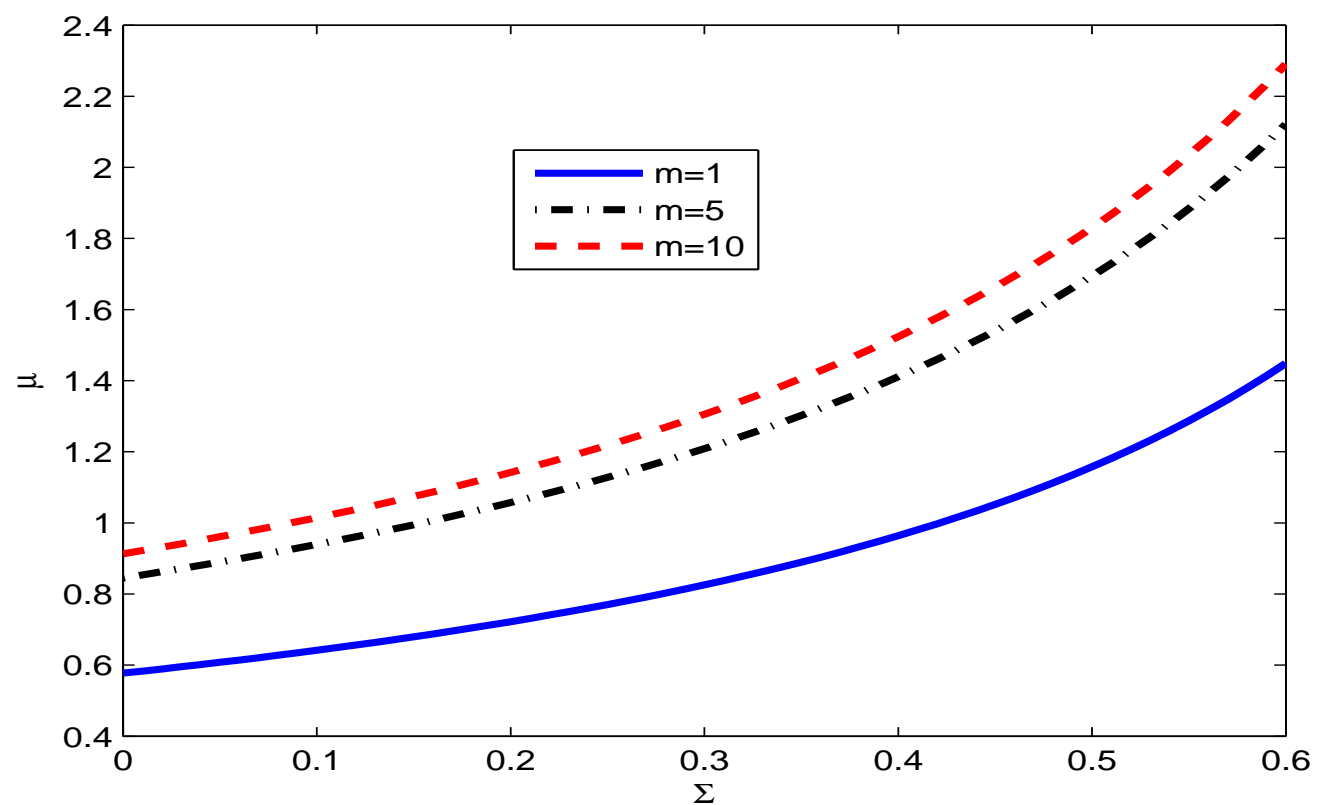

Figure 5: The Relative Volatility of Tax Change to Government Spending 


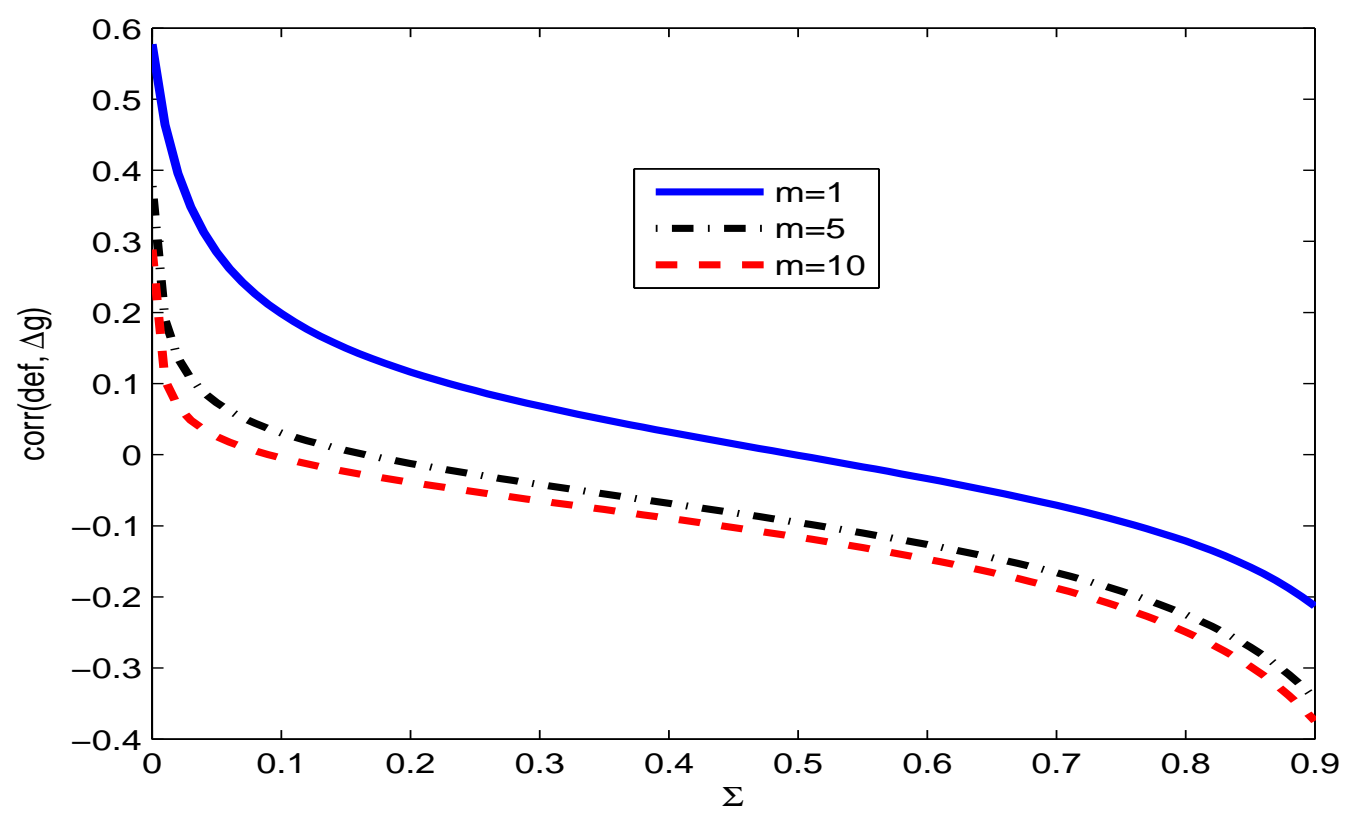

Figure 6: The Correlation between the Government Deficit and Government Spending

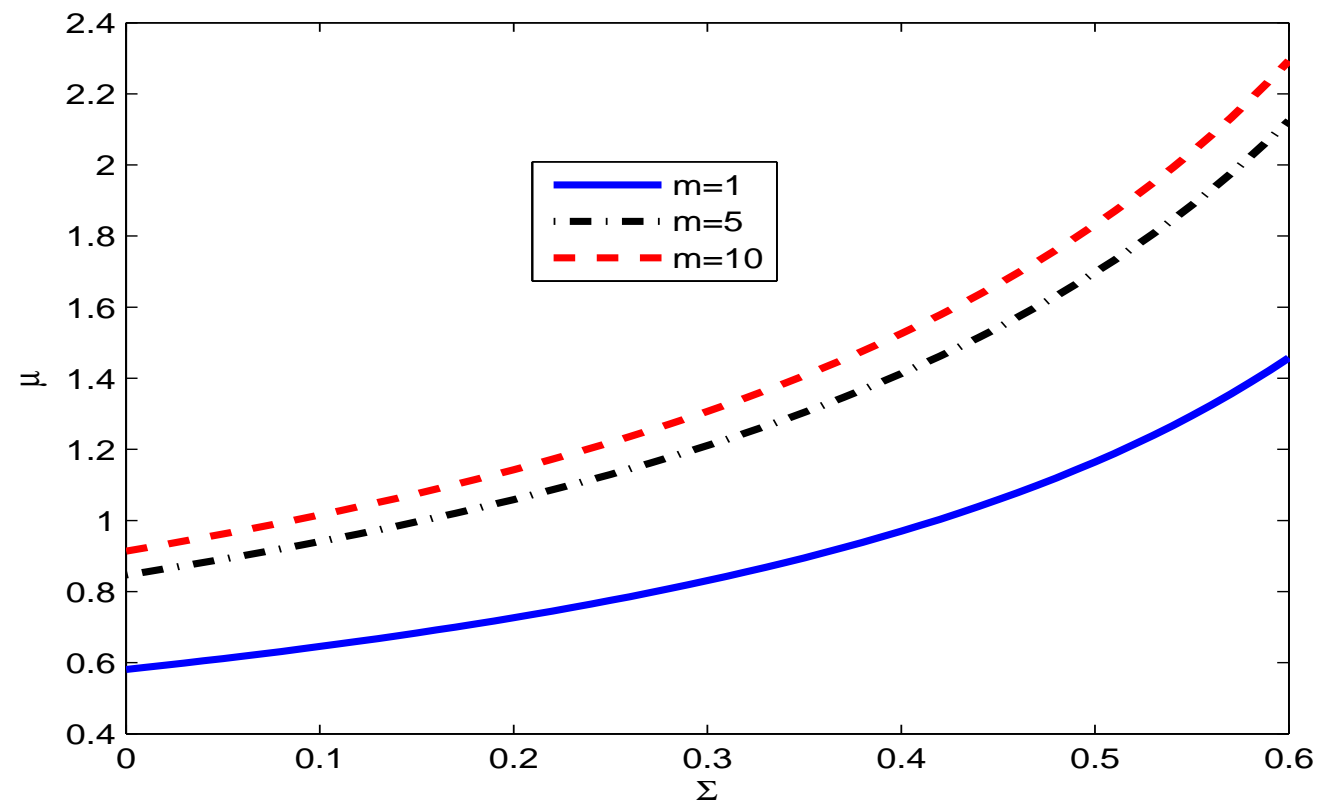

Figure 7: The Relative Volatility of Tax Change to Government Spending 


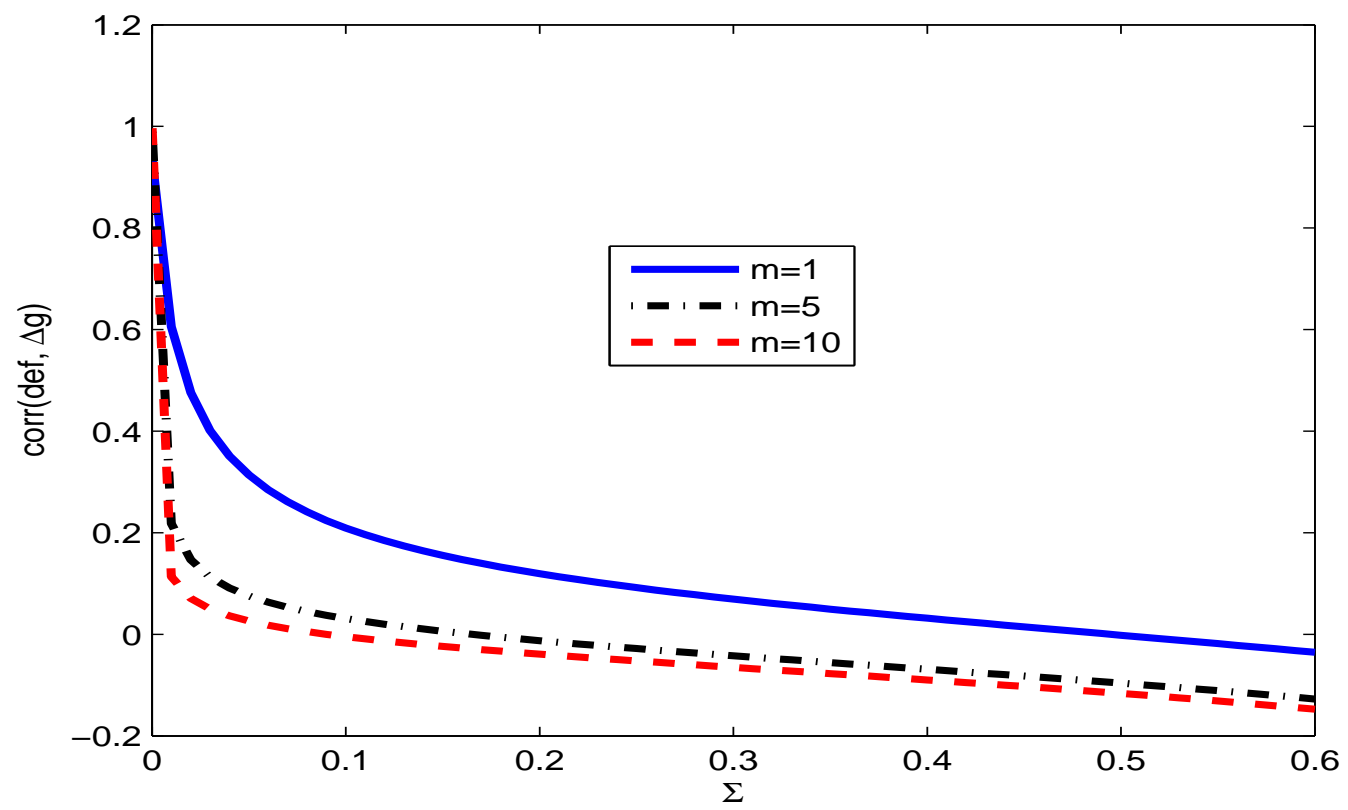

Figure 8: The Correlation between the Government Deficit and Government Spending
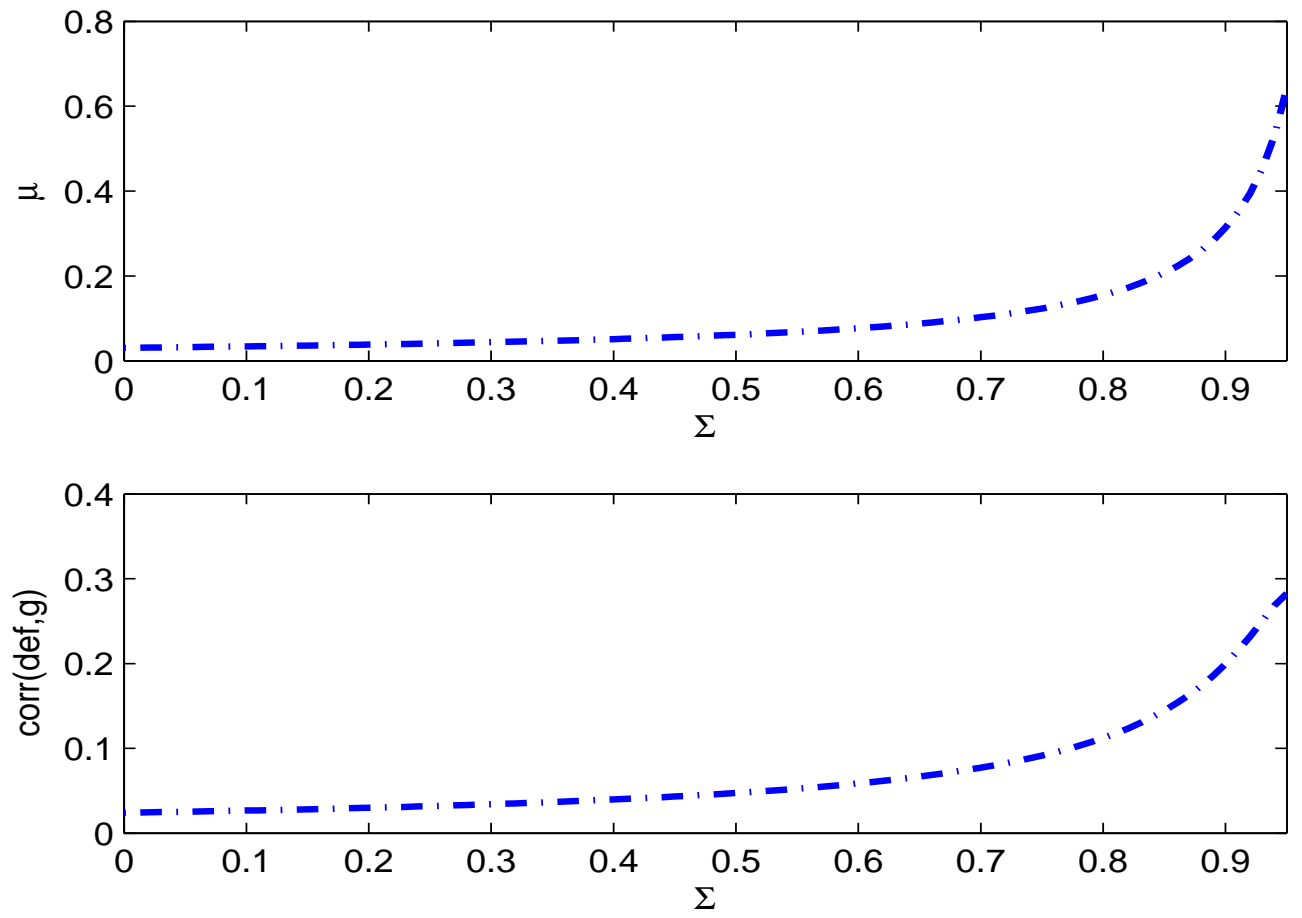

Figure 9: Relative Volatility and Correlation 


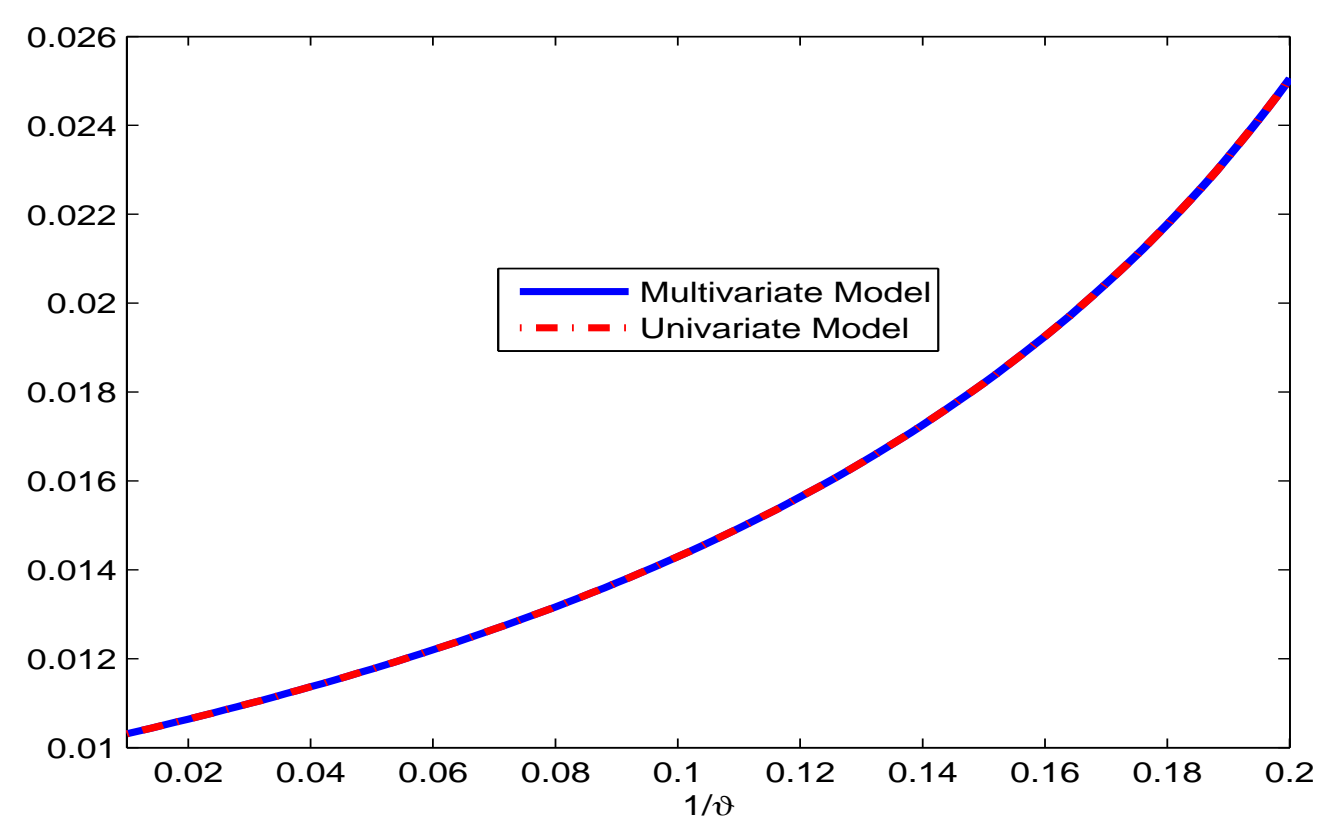

Figure 10: The Equivalence between the Multivariate and Univariate Models

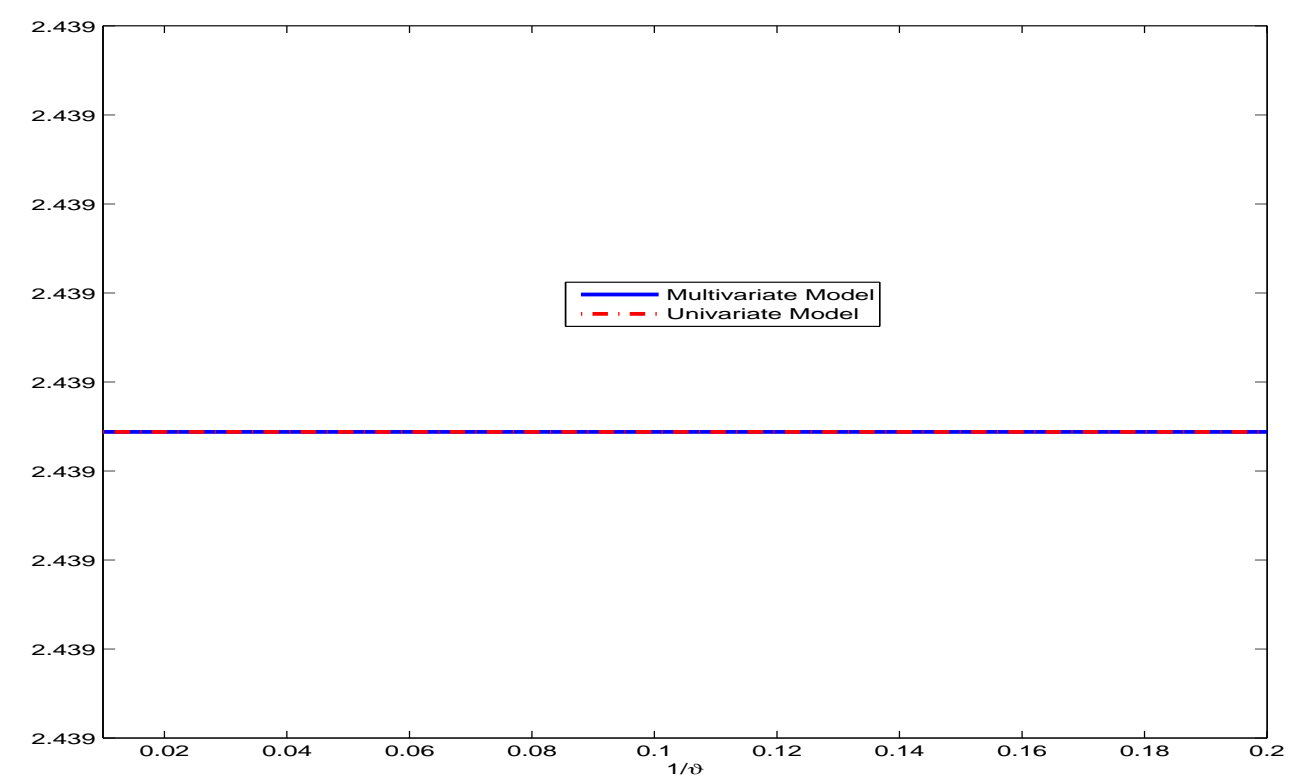

Figure 11: The Equivalence between the Multivariate and Univariate Models 
Table 1: Key Moments on Relative Volatility and Correlation

\begin{tabular}{lcc}
\hline \hline & Linear Filter & HP Filter \\
$\frac{\operatorname{sd}(\Delta \tau)}{\operatorname{sd}(\Delta g)}$ & $1.18(0.20)$ & $1.18(0.20)$ \\
$\operatorname{corr}(d e f, g)$ & $0.73(0.10)$ & $0.56(0.11)$ \\
$\operatorname{corr}(d e f, \Delta g)$ & $0.05(0.11)$ & $0.05(0.11)$ \\
$\rho_{g}$ & $0.84(0.03)$ & $0.52(0.09)$ \\
\hline \hline
\end{tabular}

Table 2: Calibration Results

\begin{tabular}{lc}
\hline \hline Variable & Value \\
\hline$\Sigma$ & 0.96 \\
$p$ & 0.10 \\
$\rho_{g}$ & 0.84 \\
$\frac{\sigma(g)}{\mu(g)}$ & 0.07 \\
$\frac{\sigma(\zeta)}{\mu(g)}$ & 0.22 \\
\hline \hline
\end{tabular}

Table 3: Model Comparison $(p=0.1)$

\begin{tabular}{lccc}
\hline \hline & Data & RE & RB \\
$\frac{\operatorname{sd}(\Delta \tau)}{\operatorname{sd}(\Delta g)}$ & 1.18 & 0.04 & 0.94 \\
$\operatorname{corr}(d e f, g)$ & 0.73 & 1.00 & 0.72 \\
\hline \hline
\end{tabular}


Table 4: Calibration: Varying The Detection Error Probability

\begin{tabular}{ccccccc}
\hline \hline & $p=0.10$ & $p=0.11$ & $p=0.12$ & $p=0.13$ & $p=0.14$ & $p=0.15$ \\
$\Sigma$ & 0.956 & 0.953 & 0.950 & 0.946 & 0.942 & 0.940 \\
\hline & $p=0.16$ & $p=0.17$ & $p=0.18$ & $p=0.19$ & $p=0.20$ & $p=0.21$ \\
$\Sigma$ & 0.937 & 0.933 & 0.928 & 0.924 & 0.918 & 0.915 \\
\hline \hline
\end{tabular}

Table 5: Robust Check: Model Comparisons

\begin{tabular}{lcccccccc}
\hline \hline & Data & $\mathrm{RE}$ & $\begin{array}{c}\mathrm{RB} \\
(p=0.10)\end{array}$ & $\begin{array}{c}\mathrm{RB} \\
(p=0.11)\end{array}$ & $\begin{array}{c}\mathrm{RB} \\
(p=0.12)\end{array}$ & $\begin{array}{c}\mathrm{RB} \\
(p=0.13)\end{array}$ & $\begin{array}{c}\mathrm{RB} \\
(p=0.14)\end{array}$ & $\begin{array}{c}\mathrm{RB} \\
(p=0.15)\end{array}$ \\
\hline$\frac{\mathrm{sd}(\Delta \tau)}{\mathrm{sd}(\Delta g)}$ & 1.18 & 0.04 & 0.94 & 0.88 & 0.82 & 0.77 & 0.71 & 0.68 \\
$\operatorname{corr}(d e f, g)$ & 0.73 & 1.00 & 0.72 & 0.73 & 0.74 & 0.76 & 0.77 & 0.78 \\
\hline & & $(p=0.16)$ & $(p=0.17)$ & $(p=0.18)$ & $(p=0.19)$ & $(p=0.20)$ & $(p=0.21)$ \\
$\frac{\operatorname{sd}(\Delta \tau)}{\operatorname{sd}(\Delta g)}$ & & 0.65 & 0.61 & 0.57 & 0.54 & 0.50 & 0.48 \\
$\operatorname{corr}(d e f, g)$ & & 0.78 & 0.80 & 0.81 & 0.81 & 0.83 & 0.83 \\
\hline \hline
\end{tabular}


Table 6: Results of the VAR Test

\begin{tabular}{l|cc|cc}
\hline \hline Model & $\lambda_{1}$ & $\lambda_{2}$ & $\chi_{W}^{2}(2)$ & p-value \\
\hline $\mathrm{RE}$ & $0.06(0.14)$ & $0.42(0.15)$ & 24.90 & 0.00 \\
$\Sigma=0.1$ & $0.06(0.13)$ & $0.44(0.15)$ & 23.60 & 0.00 \\
$\Sigma=0.2$ & $0.06(0.13)$ & $0.47(0.15)$ & 21.70 & 0.00 \\
$\Sigma=0.3$ & $0.06(0.13)$ & $0.51(0.14)$ & 19.25 & 0.00 \\
$\Sigma=0.4$ & $0.06(0.13)$ & $0.54(0.14)$ & 16.37 & 0.00 \\
$\Sigma=0.5$ & $0.06(0.13)$ & $0.58(0.13)$ & 13.32 & 0.00 \\
$\Sigma=0.6$ & $0.05(0.13)$ & $0.62(0.13)$ & 10.33 & 0.01 \\
$\Sigma=0.7$ & $0.04(0.13)$ & $0.66(0.13)$ & 7.65 & 0.02 \\
$\Sigma=0.8$ & $0.03(0.13)$ & $0.70(0.13)$ & 5.43 & 0.07 \\
$\Sigma=0.9$ & $0.02(0.14)$ & $0.73(0.14)$ & 3.74 & 0.15 \\
$\Sigma=0.96$ & $0.00(0.14)$ & $0.75(0.14)$ & 3.04 & 0.22 \\
\hline \hline
\end{tabular}

Note: $\chi_{W}^{2}(2)$ refers to the Wald statistics for the joint hypothesis test: $\lambda_{1}=0, \lambda_{2}=1$. 